\title{
Dual-Drug-Loaded Silk Fibroin/PLGA Scaffolds for Potential Bone Regeneration Applications
}

\author{
Jihang Yao $\mathbb{D},{ }^{1}$ Yilong Wang, ${ }^{2}$ Wendi $M a,{ }^{2}$ Wenying Dong, ${ }^{2}$ Mei Zhang $\left(\mathbb{D},{ }^{2}\right.$ and Dahui Sun $\left(\mathbb{D}{ }^{1}\right.$ \\ ${ }^{1}$ Norman Bethune First Hospital, Jilin University, Changchun 130021, China \\ ${ }^{2}$ Alan G. MacDiarmid Laboratory, College of Chemistry, Jilin University, Changchun 130012, China
}

Correspondence should be addressed to Mei Zhang; zhangmei@jlu.edu.cn and Dahui Sun; sundahui1971@sina.com

Received 31 January 2019; Accepted 28 May 2019; Published 16 July 2019

Academic Editor: Yu Dong

Copyright (C) 2019 Jihang Yao et al. This is an open access article distributed under the Creative Commons Attribution License, which permits unrestricted use, distribution, and reproduction in any medium, provided the original work is properly cited.

\begin{abstract}
Developing scaffold materials with excellent biocompatibility, mechanical properties, and controlled drug release properties is vital to tissue engineering. In this study, we fabricated silk fibroin (SF)/poly(lactide-co-glycolide) (PLGA) nanofiber scaffolds containing recombinant human bone morphogenetic protein 2 (rhBMP2) and dexamethasone (DXM) via coaxial electrospinning, which were used in in vitro bone formation with rat bone marrow mesenchymal stem cells (rBMSCs). An in vitro drug release study was adopted to evaluate the sustained release potential of the core-shell structured nanofibers. Furthermore, we detected the potential of the SF/PLGA nanofiber membrane in vitro. In vitro studies showed that rhBMP2 still remained active on the nanofiber membrane. In addition, the dual-drug-loaded nanofiber membrane showed an early burst release of DXM and late sustained release of rhBMP2. rhBMP2 and DXM exhibited strong osteogenic differentiation potential when they acted on rBMSCs. Therefore, the SF/PLGA nanofiber membrane loaded with rhBMP2 and DXM has great potential for the enhancement of bone regeneration.
\end{abstract}

\section{Introduction}

Although bone tissue has a self-repairing ability, when facing tumor resection, deformity, bone hypoplasia, serious fractures, and other bone injuries, the feasible way to treat bone defects is based on bone graft or implantation of allogeneic materials [1]. These treatments can restore tissue integrity and functionality, but they may also fail to provide optimal therapeutic outcomes because of the resulting poor bone quality, extensive tissue loss, or compromised regenerative capacity.

One strategy for repairing or replacing bone is to design bone tissue by implanting scaffolds with a combination of cells and bioactive molecules [2-4]. The ideal scaffold should have the following properties: biocompatibility, biodegradability, and excellent mechanical strength. The scaffold should promote early mineralization and support the formation of new bone $[5,6]$. At the same time, it allows the replacement of the old organization. In other words, scaffolds should have a physical structure and a chemical composition similar to natural bone.

As one of the most commonly used nanofiber synthetic materials in tissue engineering, PLGA has been approved for clinical use by the FDA in the United States. PLGA has been widely used in medical applications such as suture materials, implants, and controlled drug delivery systems [7-9]. However, like other biopolymers, PLGA has poor hydrophilicity. Its absorption rate for proteins is low, and there is no natural cell recognition site on the surface of PLGA, resulting in the poor affinity of cells. [10] In order to improve its surface properties, there are several ways to modify it. The development of composite materials may provide a way forward. Silk fibroin (SF) is a natural biological material with remarkable biomedical and mechanical properties, which is conducive to a wide range of bone tissue engineering applications. It can be fabricated into different scaffolds, combined 
with other biomaterials to form composites and chemical modifications, provide a unique toolbox, and allow silk fiber scaffolds to be suitable for specific applications [11-14].

In recent years, biodegradable nanofiber technology has been widely used. Electrospinning is a relatively mature method for the preparation of nanofibers at present. The prepared membrane fibers have a small diameter and a large specific surface area and are extremely similar to the natural extracellular matrix (ECM) in morphology and structure, which is conducive to cell adhesion, proliferation, and activity. At the same time, the nanofiber also has a high specific surface area, which is conducive to the adsorption and release of active cells and growth factors, and can significantly improve the biological properties of composite materials. In addition, the preparation of the nanofiber structure is simple and the fiber structure is adjustable, which can meet the needs of different tissue engineering applications. Studies in vivo and in vitro have shown that the nanofiber structure can absorb different growth factors and play a synergistic role in tissue growth. Coaxial electrospinning is a major advance in technology in the development of electrospinning [15-17]. The fibers prepared by this method not only have a high specific surface area and good mechanical properties, but they also have a unique core-shell structure, which can wrap biological molecules into nanofibers, maintain the activity of biological molecules, and achieve the purpose of carrying and sustained release. It can enhance the biological properties of materials and has broad application prospects in the field of tissue engineering.

There are several types of bone growth factors, including bone morphogenetic growth factors (BMP), fibroblast growth factor (FGF), platelet-derived growth factor (PDGF), vascular endothelial growth factor (VEGF), and insulin-like growth factor (IGF). Among all these growth factors, BMP is considered to be the most effective way to format bone tissue during embryonic development, growth, and healing and throughout adulthood [18, 19]. An increasing number of reports tested the in vivo application and biological foundation when BMP is used in bone defects [20-22]. Dexamethasone (DXM) is an effective form of the steroid glucocorticoid that has been widely used in various medical and biological applications [23]. Previous studies reported that DXM can induce osteoporosis and even pathological fracture, while DXM in vitro promotes osteoblast differentiation and bone mineralization [24].

In this study, we describe a new scaffold where the controlled release of two bioactive factors (rhBMP2 and DXM) was achieved by using an SF/PLGA core-shell through coaxial electrospinning. The core-shell structure of the nanofiber was analyzed by different characterization methods. In vitro drug release studies were carried out to study the potential of nanofiber scaffolds as drug delivery vectors. Osteogenic differentiation and osteogenesis of this scaffold were evaluated by measuring the adhesion, proliferation, and osteogenic differentiation of rBMSCs including ALP activity and extracellular matrix calcium content. The purpose of this study is to prepare ideal core-shell nanofibers from a natural biomaterial (silk fibroin), with the controlled release capability of rhBMP2 and DXM, in order to promote the osteogenic expression of bone marrow mesenchymal stem cells for bone tissue engineering and explore the potential of natural biomaterials as bone tissue engineering materials.

\section{Materials and Method}

2.1. Animals. All animal experiments were performed in accordance with the guidelines of the Chinese Experimental Animal Management Association for the Care and Use of Laboratory Animals. Three-week-old male Sprague-Dawley rats (Liaoning Chang Sheng Biotechnology Co. Ltd., Liaoning, China) were used for in vitro experiments. Animals were sacrificed by exposure to $100 \% \mathrm{CO}_{2}$ followed by cervical dislocation.

2.2. Materials. Silk fibroin $\left(M_{w}=100 \mathrm{kDa}\right)$ was purchased from Suzhou Simatech Co. Ltd. (Suzhou, China), and PLGA $\left(M_{w}=1.8 \times 10^{5} \mathrm{Da}\right)$ was purchased from Jinan Daigang Biotechnology Co. Ltd. (Jinan, China). rhBMP2 $\left(M_{w}=12.9 \mathrm{kDa}\right)$ was obtained from Beijing Wish Biotechnology Co. Ltd. (Beijing, China). DXM $\left(M_{w}=392.2 \mathrm{Da}\right)$ and bovine serum albumin (BSA) were purchased from Sigma-Aldrich (St. Louis, MO, USA). The solvent 1,1,1,3,3,3,-hexafluoro-2-propanol (HFIP) that was used to dissolve PLGA, SF, and their blends was sourced from Energy Chemical Co. Ltd. (Tianjin, China). CCK-8 was purchased from Beyotime Biotechnology Co. Ltd. (Shanghai, China). ARS solutions were purchased from Sigma-Aldrich (Shanghai, China). The ALP staining kit (GOMORI, KGA353) and ALP quantitative test (KGT043) were purchased from KeyGen Biotech Co. Ltd. (Nanjing, China). Phosphate-buffered saline (PBS), ketamine, xylazine, and isopropanol were purchased from SigmaAldrich (USA).

2.3. Fabrication of rhBMP2- and DXM-Loaded SF/PLGA Nanocomposite Scaffolds. rhBMP2- and DXM-loaded SF/PLGA nanocomposite scaffolds were prepared using a coaxial electrospray method (Figure 1). The spray nozzle used in this experiment consists of an outer needle of 18 gauge and an inner needle of 25 gauge. Initially, SF, PLGA, SF-DXM, and PLGA-rhBMP2 were dissolved in HFIP. The SF-DXM solutions were prepared by dissolving $148.5 \mathrm{mg}$ of $\mathrm{SF}$ and $1.5 \mathrm{mg}$ of DXM in $1.5 \mathrm{~mL}$ of HFIP. A solution (10 wt\%) containing only SF and a solution (10wt\%) containing only PLGA were also prepared. The solutions were stirred magnetically for $4 \mathrm{~h}$ at room temperature. The PLGA-rhBMP2 solution was prepared by dissolving $\operatorname{rhBMP} 2(5,15$, and $25 \mu \mathrm{g} / \mathrm{mL}$, stabilized by $1 \%$ BSA) into the PLGA solution before electrospinning. PLGA or the PLGA-rhBMP2 solution was used in the core solution at a flow rate of $0.4 \mathrm{~mL} / \mathrm{h}$, and SF and the SF-DXM solution was used in the shell solution at a flow rate of $1.2 \mathrm{~mL} / \mathrm{h}$.

The high voltage of the coaxial electrospinning techniques was adjusted to $20 \mathrm{kV}$. A piece of aluminum foil was placed $120 \mathrm{~mm}$ directly below the needle to act as a collector. Electrospinning fibrous scaffolds were immersed in $90 \%$ $(v / v)$ methanol for $30 \mathrm{~min}$ to induce an amorphous regionto- $\beta$-sheet transition of SF. Subsequently, the methanol- 


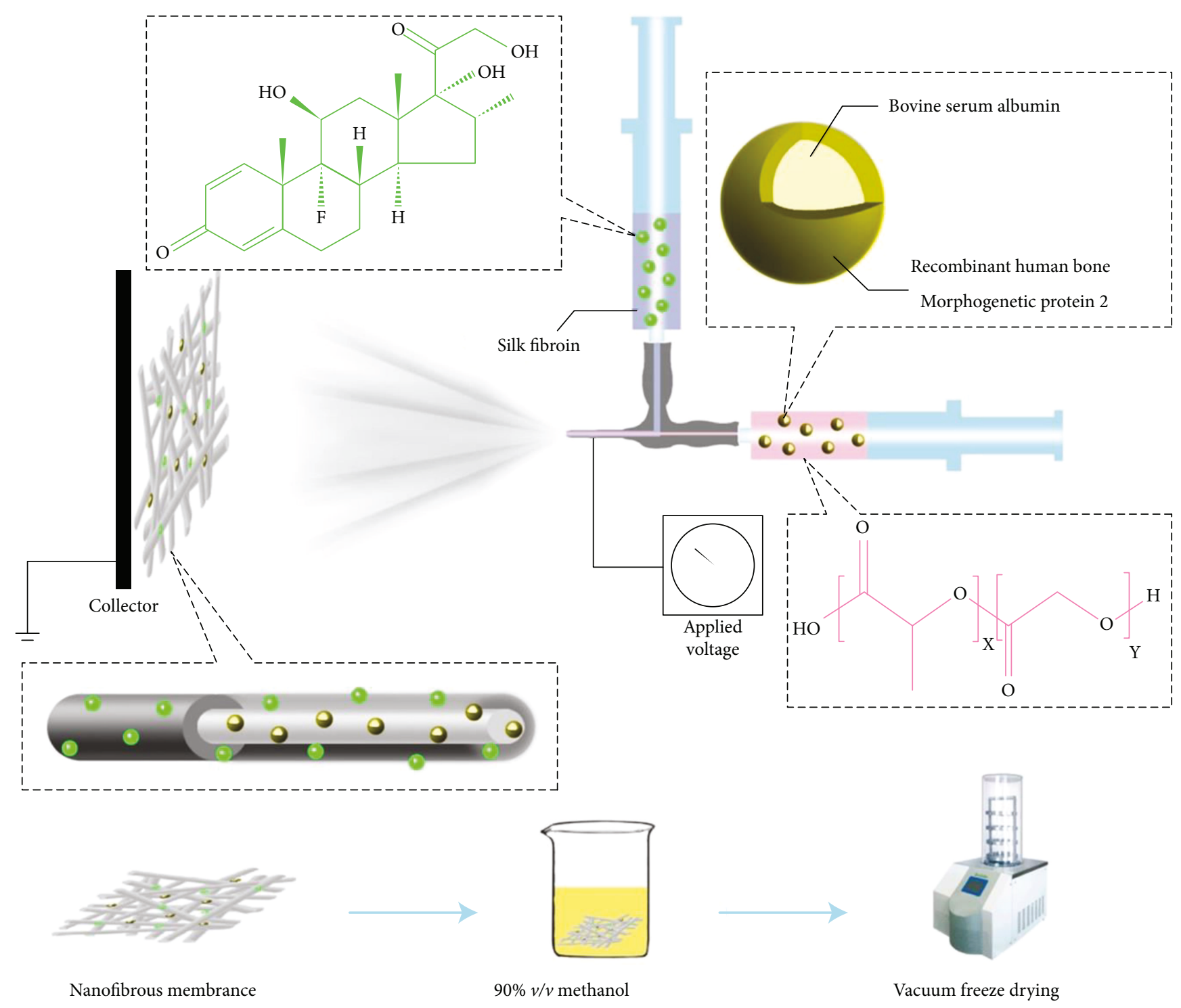

FIgURE 1: Schematic illustration of the scaffold fabrication processes.

treated scaffolds were freeze-dried at $-30^{\circ} \mathrm{C}$ to remove the solvents and stored at $-20^{\circ} \mathrm{C}$ for further analysis.

\subsection{Environmental Scanning Electron Microscopy (ESEM)} and Transmission Electron Microscopy (TEM). The morphology of the nanofibers was observed by using ESEM (XL-30, FEI Co. Ltd., USA) at an accelerating voltage of $15 \mathrm{kV}$ after taking a piece of aluminum foil sputter-coated with gold for 90 s. The SEM micrographs were analyzed by Nano Measurer 1.2 to determine the average diameter and examine the uniformity of the nanofibers.

The core-shell structure of the nanofibers was observed by TEM (H-7650, Hitachi Co. Ltd., Japan) at an accelerating voltage of $110 \mathrm{kV}$. The nanofibers were collected by the 400 mesh copper network.

2.5. Contact Angle Measurement. The water contact angle of the fibrous membrane was measured by the XG-CAM (Shanghai Xuanyi Creative Analysis Industrial Manufactur- ing Co. Ltd.). Experiments were performed by dropping deionized water on a piece of flat nanofiber scaffolds. Each sample was tested three times on different parts of the membrane. The contact angle was read at the 30th second.

2.6. Fourier-Transform Infrared (FTIR) and X-Ray Photoelectron Spectroscopy (XPS). Chemical analysis of experiments was performed by FTIR spectroscopy at the range of $4000-400 \mathrm{~cm}^{-1}$. FTIR spectra of the PLGA/SF nanofibrous scaffolds were obtained by a Nicolet spectrometer system (System 2000, PerkinElmer) with a DTGS KBr detector. $1 \mathrm{mg}$ of the dried sample was mixed with $200 \mathrm{mg}$ of $\mathrm{KBr}$ and compressed into pellets. The element composition of the samples was analyzed by XPS using an ESCALAB 250 spectrometer with a mono $\mathrm{X}$-ray $\mathrm{Al} \mathrm{K} \alpha$ excitation source $(1486.6 \mathrm{eV})$.

2.7. Mechanical Performance Test. The mechanical property of the scaffolds was tested using a universal material testing 
machine (TestResources, USA). Eight groups of rectangular shapes $(10 \mathrm{~mm} \times 50 \mathrm{~mm})$ were prepared and measured at a speed of $10 \mathrm{~mm} / \mathrm{min}$ at room temperatures. Before the tensile test, the thickness of each fibrous membrane was measured by a spiral micrometer. Samples of each group were tested three times on different parts of the membranes.

2.8. In Vitro Drug Release. A certain amount of nanofiber mats was weighed in a $10 \mathrm{~mL}$ tube, and $3 \mathrm{~mL}$ phosphatebuffered saline (PBS, $\mathrm{pH}=7.4$ ) solution was added to the tube. The tube was placed in a constant temperature water bath oscillator for 21 days. The water temperature was set to $37^{\circ} \mathrm{C}$, and the rotate speed was $100 \mathrm{rpm}$. After certain time intervals, $2 \mathrm{~mL}$ samples were extracted from the release medium, and $2 \mathrm{~mL}$ fresh media was added to the release medium. The samples were collected and frozen at $-20^{\circ} \mathrm{C}$ until analyzed. The collected supernatant was tested by an rhBMP2 ELISA kit. Data were accumulated by measuring adsorption at $450 \mathrm{~nm}$ using a microplate reader (Bio-Rad iMark 14071). DXM concentration was measured with an ultraviolet spectrophotometer (UV-2450, Japan). Each experiment was repeated 3 times to get the average value.

2.9. Cell Isolation and Culture. BMSCs were obtained by the aspiration of bone marrow from 3-week-old male SpragueDawley rats [25]. Purified BMSCs were cultured in DMEM medium containing $10 \%$ fetal bovine serum. Three generations of rat BMSCs were used for analysis and were removed from the culture by trypsinization.

The electrospinning nanofibers collected on $15 \mathrm{~mm}$ diameter coverslips were placed in a 24 -well plate with a stainless steel ring to prevent the nanofibers from lifting. After having been sterilized with Cobalt- $60,200 \mu \mathrm{L}$ of the DMEM medium containing $10 \%$ fetal bovine serum was added to preinfiltrate for 12 hours. Three generations of rat BMSCs were made into a cell suspension. Cell concentration was adjusted to $2.5 \times 10^{4} / \mathrm{mL}$. $200 \mu \mathrm{L}$ of cell suspension was added into each well. After 4 hours of incubation in a cell incubator, the culture medium was slowly increased to $1 \mathrm{~mL}$ along the chemical wall and then continued to culture. The culture medium was replaced every 2 days.

Cell proliferation was evaluated by using the CCK- 8 assay. After culturing the cells for a period of 1,4 , and 7 days, $200 \mu \mathrm{L}$ of CCK-8 reagent was added to each well and incubated for $2 \mathrm{~h}$ at $37^{\circ} \mathrm{C}$ using a $5 \% \mathrm{CO}_{2}$ incubator. Thereafter, the samples were pipetted into 96-well plates and the absorbance was measured at $450 \mathrm{~nm}$ using a microplate reader.

The cell morphology of rBMSCs was analyzed by using ESEM (S-3400N, Hitachi Co. Ltd., Japan). After 3 days of seeding cells on the scaffolds, the media was removed, the cells were washed three times with PBS for $15 \mathrm{~min}$, and then the samples were fixed with $4 \%$ glutaraldehyde for $2 \mathrm{~h}$ under $4^{\circ} \mathrm{C}$. The scaffolds were rinsed in distilled water for $15 \mathrm{~min}$. Then, they were dehydrated by ethanol gradient, dried by dryer, sprayed with gold, and observed with a scanning electron microscope at an accelerating voltage of $10 \mathrm{kV}$. Confocal laser scanning microscopy is also used to observe cell morphology and proliferation. After 4 days of culture, the media was removed and the cells were washed three times with PBS for 10 min each time and fixed with $4 \%$ polyformaldehyde for $20 \mathrm{~min}$; then, the cells were washed twice with PBS, stained with $5 \mathrm{ug} / \mathrm{mL}$ phalloidin at room temperature for $30 \mathrm{~min}$, and washed twice with PBS. The nucleus was stained with DAPI for $10 \mathrm{~min}$ and observed by confocal laser scanning microscopy (CLSM, Leica TCS SP8, Germany).

2.10. Alkaline Phosphatase Activity. To stain the colonies positive for ALP after 7 days, the cells were fixed in $4 \%$ formaldehyde for $10 \mathrm{~min}$ and then stained for $30 \mathrm{~min}$ at room temperature; finally, the cells were washed three times with distilled water for $15 \mathrm{~min}$. The numbers and total areas of positive colonies were measured by image analysis using ImageJ software.

2.11. Mineralization of Osteogenic Cells. On the 14th day, different electrospinning scaffolds seeded with rBMSCs were washed three times in PBS and fixed in 4\% paraformaldehyde for $30 \mathrm{~min}$ at $4^{\circ} \mathrm{C}$. Then, they were washed three times with distilled water and stained with $1 \%$ ARS for 20 min at room temperature. After washing three times with distilled water, the scaffolds were observed under an inverted optical microscope and images were taken using an optical microscope.

2.12. Statistical Analysis. All experiments were performed in triplicate, and statistical analysis was performed using SPSS software (version 13.0; SPSS Inc., Chicago, IL, USA). All data are reported as mean \pm standard deviations. The significance level was set to $p<0.05$.

\section{Results}

3.1. Morphology of Nanofibrous Scaffolds. All the nanofibers produced in this study were ground to microscale and nanoscale, as shown in Figure 2. In addition, all seven groups of nanofibers showed relatively uniform and bead-free morphology. Compared to other six groups, SF/PLGA showed the smallest diameter $(0.37 \pm 0.094 \mu \mathrm{m})$ with circular fiber morphology (Figure 2(a)). The average diameter of the SF/PLGA-rhBMP2 nanofibers increased with the addition of rhBMP2, and the fibers tend to flatten (Figures 2(b)$2(\mathrm{~d}))$. With the addition of DXM, the average diameters of the SF-DXM/PLGA-rhBMP2 nanofibers decreased and the fibers tend to be more circular (Figures $2(\mathrm{e})-2(\mathrm{f})$ ).

Figure $2(\mathrm{a} 1, \mathrm{~d} 1, \mathrm{~g} 1)$ also showed the representative TEM micrographs of the coaxial SF/PLGA, SF/PLGA-rhBMP2, and SF-DXM/PLGA-rhBMP2 nanofibers. There was a clear interface between the core and shell of the nanofibers. In addition, all nanofibers exhibited a continuous and homogeneous core-shell structure. The core-shell structure of the nanofiber was developed and assessed by transmission electron microscopy (H-7650, Hitachi Co. Ltd., Japan) and confocal laser scanning microscopy (CLSM; LSM 780, Carl Zeiss, Jena, Germany). The presence of the core-shell structure of the nanofiber was apparent, and the boundary between the two layers was sharp (Figure 1(a)). The outer diameters of the representative coaxial nanofibers were $0.298,0.67$, and $0.582 \mu \mathrm{m}$ accompanied with the inner diameters of 0.0785 , 0.136 , and $0.121 \mu \mathrm{m}$ (Figure $2(\mathrm{~h})$ ), respectively, which was in accordance with the SEM results. Figure 2(i) also shows 
(a1)

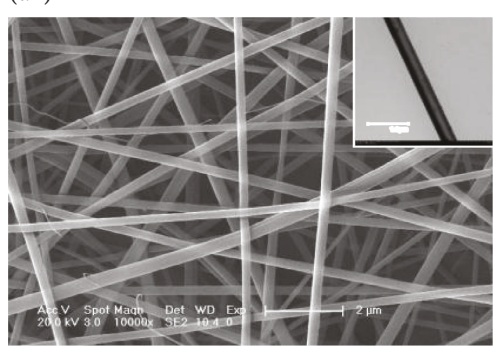

(a2)

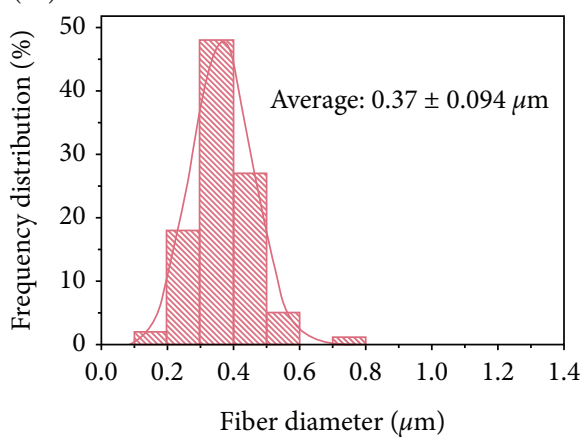

(a)

(b2)
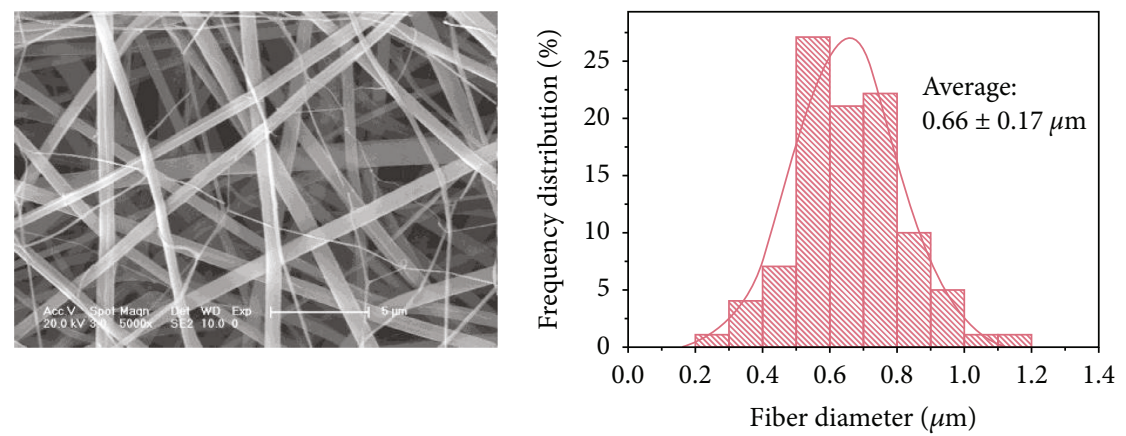

(b)

(c2)

(c1)
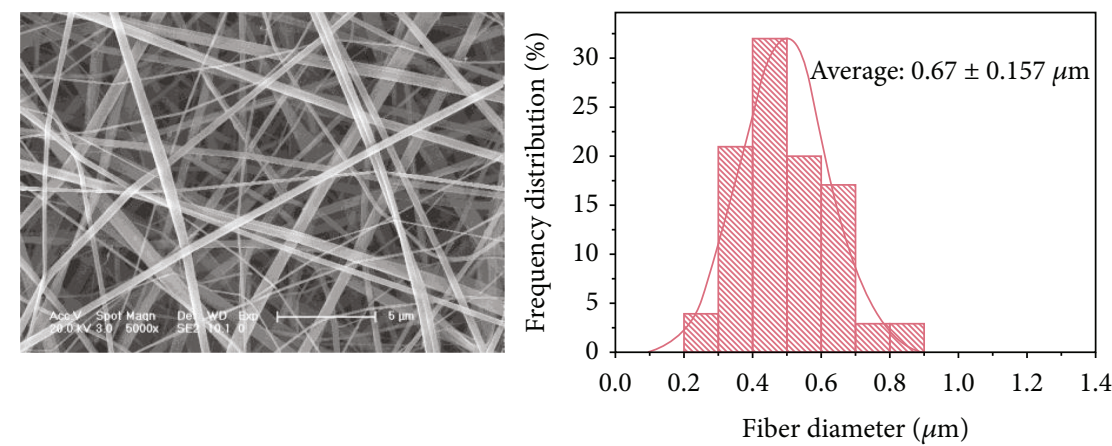

(c)

(d2)
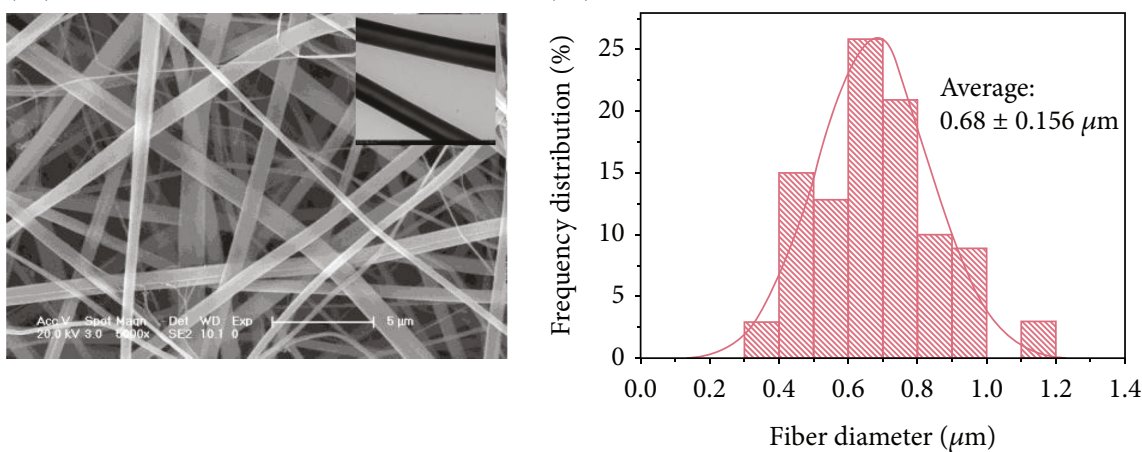

(d)

Figure 2: Continued. 
(e1)

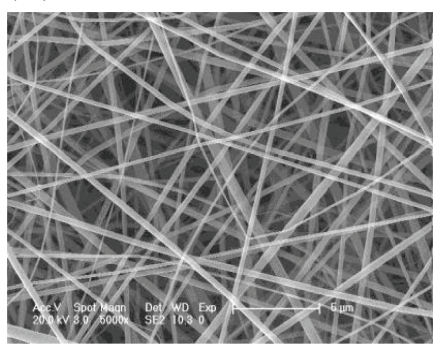

(e2)

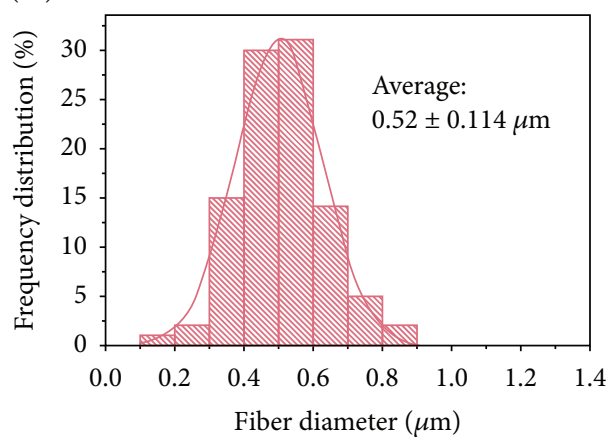

(e)

(f2)
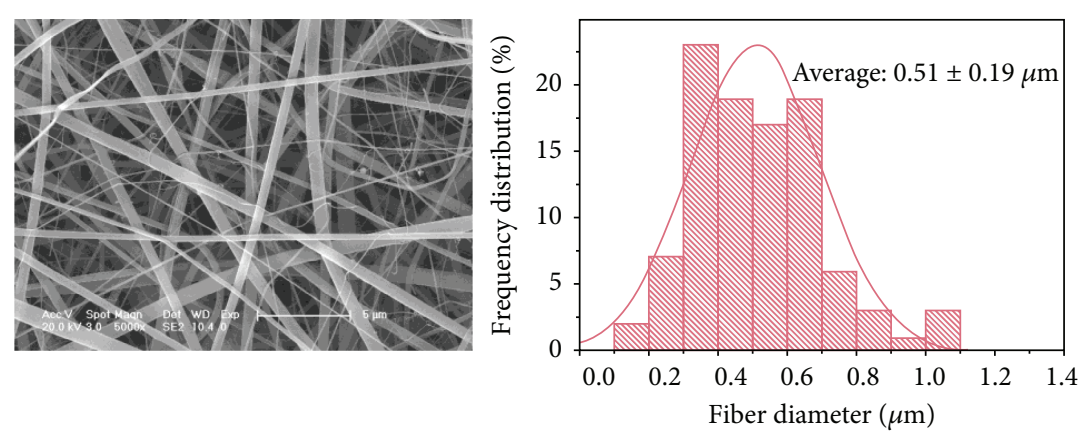

(f)

(g2)

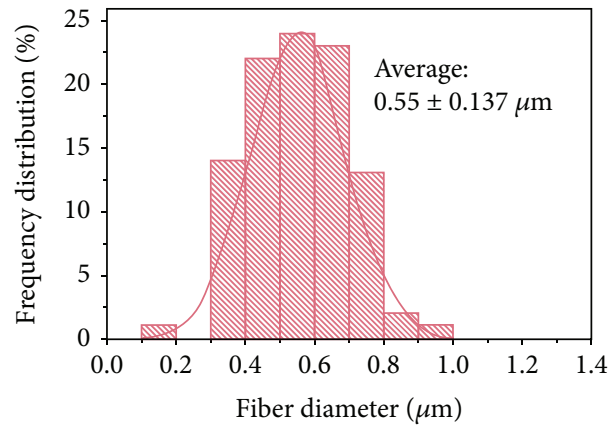

(g)

(h2)

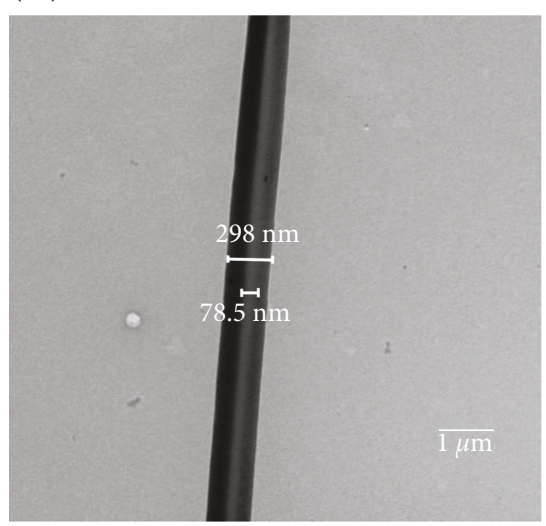

(h3)

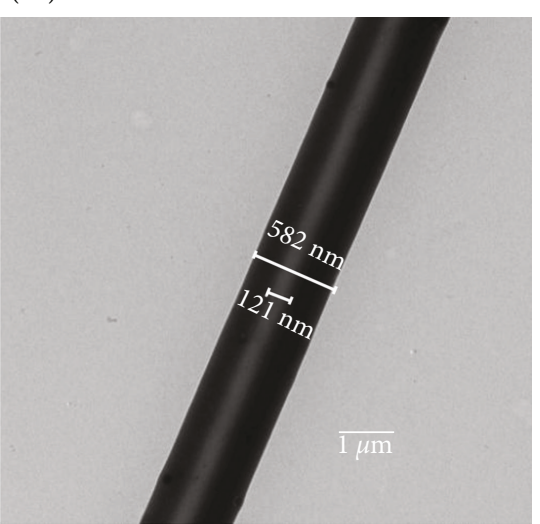

(h)

Figure 2: Continued. 
(i1)

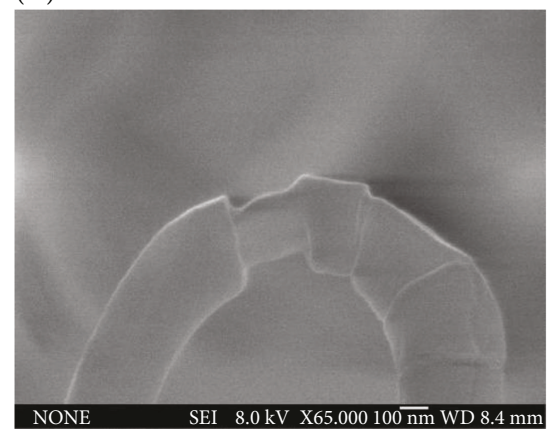

(j1)

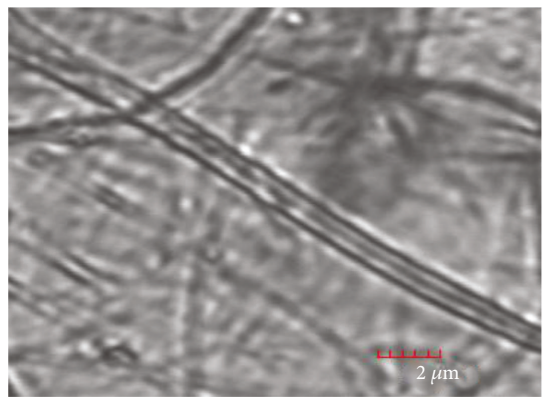

(i2)

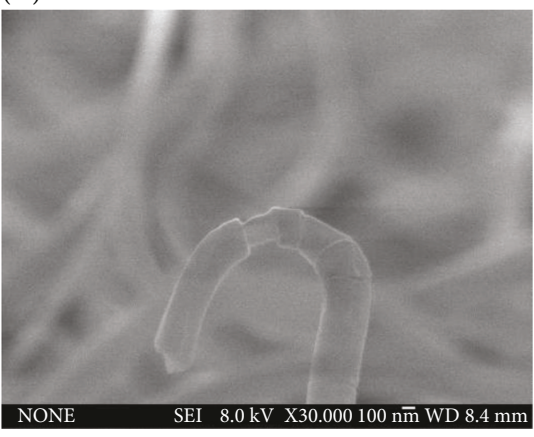

(i)

(j2)

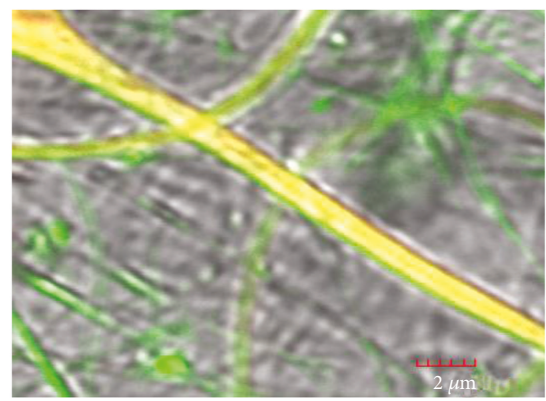

(i3)

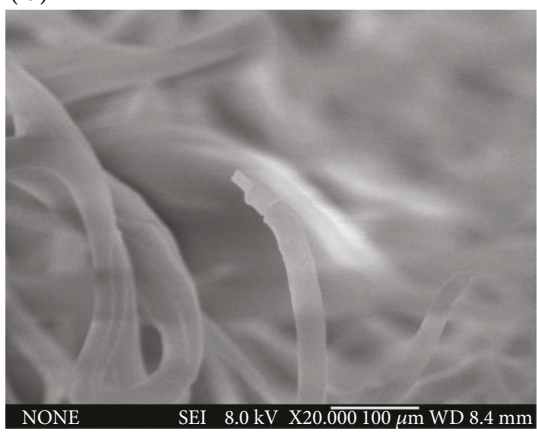

(j3)

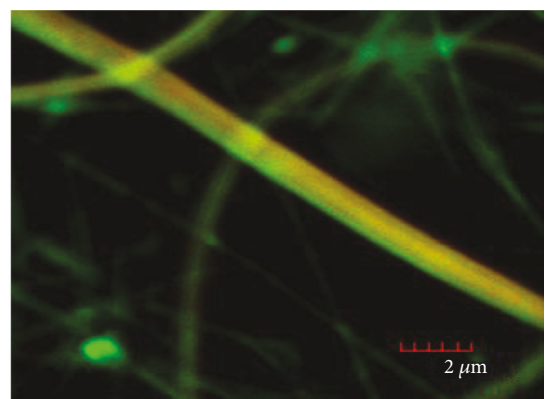

(j)

Figure 2: SEM images of nanofibers (1) and diameter distribution (2): (a and i) SF/PLGA, (b) SF/PLGA-rhBMP2 (5 $\mu$ g), (c) SF/PLGArhBMP2 $(10 \mu \mathrm{g}),(\mathrm{d})$ SF/PLGA-rhBMP2 $(25 \mu \mathrm{g})$, (e) SF-DXM/PLGA-rhBMP2 (5 $\mu \mathrm{g})$, (f) SF-DXM/PLGA-rhBMP2 (10 $\mu \mathrm{g})$, and (g) SFDXM/PLGA-rhBMP2 $(25 \mu \mathrm{g})$; scale bar $=5 \mu \mathrm{m}$. TEM images of coaxial nanofibers: (a1 and h1) SF/PLGA, (d1 and h2) SF/PLGArhBMP2 $(25 \mu \mathrm{g}),(\mathrm{g} 1$ and $\mathrm{h} 3)$ and SF-DXM/PLGA-rhBMP2 $(25 \mu \mathrm{g})$; scalebar $=1 \mu \mathrm{m}$. CLSM images of coaxial nanofibers: (j) SF/PLGA; scale bar $=2 \mu \mathrm{m}$.

that there are obvious interfaces between the outer and inner layers. Furthermore, the inner core of the nanofiber and the outer core of the nanofiber was incorporated, respectively, with rhodamine $\mathrm{B}$ and sodium fluorescein which was analyzed through CLSM. The inner core emitted a distinct red fluorescence, and the outer core emitted a green fluorescence (Figure 2(j)).

3.2. Contact Angle Measurement. As shown in Figure 3, pure PLGA scaffolds showed hydrophobicity $\left(143.4 \pm 2.1^{\circ}\right)$, while pure SF scaffolds showed hydrophilicity $\left(74.3 \pm 0.8^{\circ}\right)$. The coaxial SF/PLGA scaffolds exhibited similarity in hydrophilicity $\left(67.9 \pm 7.45^{\circ}\right)$ due to the outer shell composite. In addition, the addition of DXM and rhBMP2 also had little effect on the hydrophilicities of SF/PLGA-rhBMP2 $\left(66.0 \pm 1.69^{\circ}\right)$ and SF-DXM/PLGA-rhBMP2 $\left(68.7 \pm 2.98^{\circ}\right)$.

3.3. FTIR and XPS. As shown in Figure 4, strong absorption peaks at 1759,1182 , and $1132 \mathrm{~cm}^{-1}$ indicated the stretching vibration of the $\mathrm{C}=\mathrm{O}$ bond, the $\mathrm{C}-\mathrm{O}-\mathrm{C}$ bond, and the $\mathrm{C}-\mathrm{O}$ single bond, respectively. In addition, the antisymmetric bending vibration absorption peak of methyl was located near $1455 \mathrm{~cm}^{-1}$. The absorption peaks of pure SF scaffolds were located at $1530 \mathrm{~cm}^{-1}$ (random coils) and $1238 \mathrm{~cm}^{-1}$ ( $\alpha$-helix). Most conformations were random coils, while small amounts were $\alpha$-helix structures. Coaxial SF/PLGA electrospun fibers showed not only the characteristic

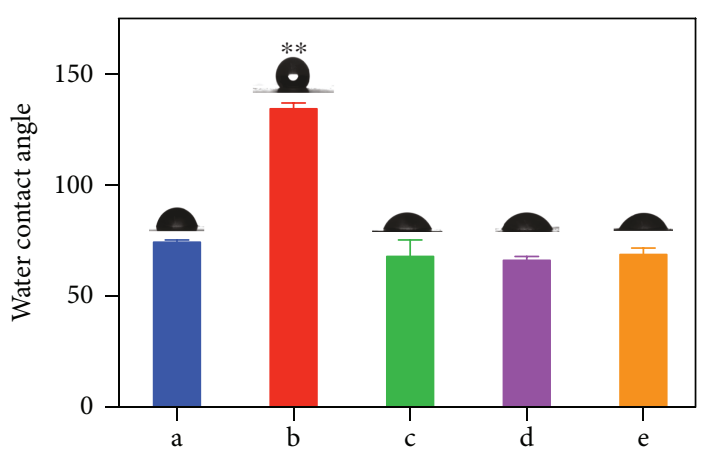

FIGURE 3: Water contact angle of the composite nanofiber membrane: (a) SF, (b) PLGA, (c) SF/PLGA, (d) SF/PLGArhBMP2, and (e) SF-DXM/PLGA-rhBMP2; ${ }^{* *} p<0.01$. The content of rhBMP2 is $25 \mu \mathrm{g}$.

absorption peaks of PLGA, but also two characteristic absorption peaks of SF. The FTIR spectrum of SF treated with methanol shows that the absorption peaks were mainly located at $1655 \mathrm{~cm}^{-1}$ (irregular group) and $1519 \mathrm{~cm}^{-1}(\beta$-fold), which indicated that the conformations are mainly composed of the $\beta$-fold structure and a small amount of random coil structure.

XPS is used to verify whether DXM is successfully loaded into the fibrous membrane, because the $F$ element is the characteristic element of DXM. Through comparison 


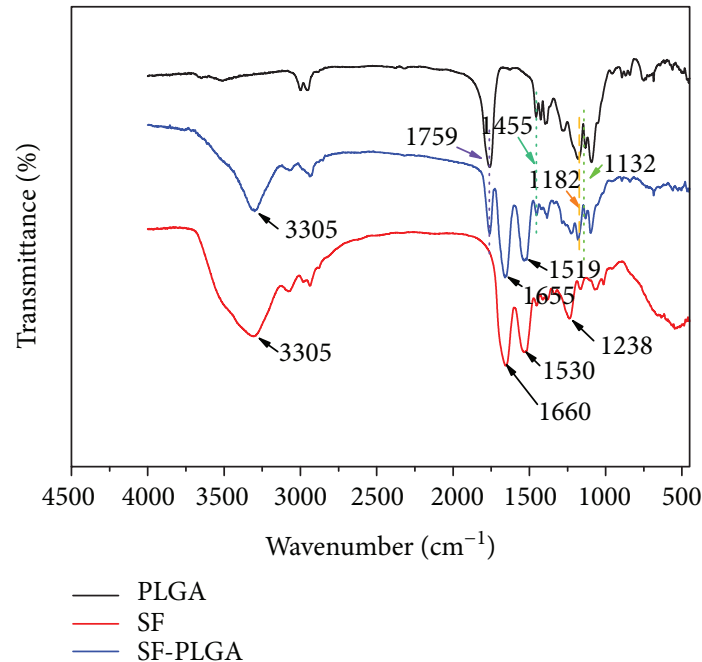

FIGURE 4: FTIR spectra of PLGA, SF, and SF/PLGA (treated by $90 \%$ $(v / v)$ methanol) membranes.

of the XPS spectrum of SF/PLGA (Figure 5(a)) with that of SF-DXM/PLGA (Figure 5(b)), the $F$ peak at $689 \mathrm{eV}$ (Figure 5(b)) indicated that DXM was successfully loaded into nanofibers.

3.4. Mechanical Performance Test. The tensile stress-strain curves of different groups of nanofibers were characterized. The results showed that SF/PLGA nanofibers had high tensile strength, Young's modulus, and elongation at break (Figure 6). The tensile strength of SF-DXM/PLGA increased slightly compared to that of SF/PLGA, and it was similar with that of SF/PLGA-rhBMP2 and SF-DXM/PLGA-rhBMP2. The elongation at break of SF-DXM/PLGA was similar to that of pure nanofibers. With the addition of rhBMP2, the tensile strength, Young's modulus, and elongation at break of the nanofiber membrane significantly reduced, while those of SF-DXM/PLGA-rhBMP2 are the lowest, which illustrates that the addition of rhBMP2 reduces the tensile strength and elongation at break of the nanofibers.

3.5. In Vitro Drug Release. As shown in Figure 7, DXM in the SF/PLGA nanofiber membrane showed an abrupt release in the initial $12 \mathrm{~h}$ and a continuous slow release for 21 days. Accumulated DXM releases in the SF/PLGA nanofiber membrane in 21 days ranked from highest to lowest are as follows: $80.7,70.02,69.5$, and $58.13 \mu \mathrm{g}$. It can be seen that the release rate of DXM increased with the addition of rhBMP2, and it was positively correlated with the concentration of rhBMP2. On the other hand, DXM showed an abrupt increase in the initial $12 \mathrm{~h}$, reaching 62.1, 59.3, 49.03 , and $47.06 \mu \mathrm{g}$ in SF-DXM/PLGA-rhBMP2 $(25 \mu \mathrm{g})$, SF-DXM/PLGA-rhBMP2 $(10 \mu \mathrm{g})$, SF-DXM/PLGA-rhBMP2 $(5 \mu \mathrm{g})$, and SF-DXM/PLGA, respectively.

Accumulated rhBMP2 release in 21 days in SF-DXM/ PLGA-rhBMP2 $(5 \mu \mathrm{g})$, SF-DXM/PLGA-rhBMP2 $(10 \mu \mathrm{g})$, and SF-DXM/PLGA-rhBMP2 $(25 \mu \mathrm{g})$ and in SF/PLGArhBMP $(5 \mu \mathrm{g})$, SF/PLGA-rhBMP2 $(10 \mu \mathrm{g})$, and SF/PLGArhBMP2 $(25 \mu \mathrm{g})$ were $30.84 \%, 18.46 \%$, and $9.2 \%$ and $21.9 \%$,
$18.55 \%$, and $11.71 \%$, respectively. It indicated that rhBMP2 was encapsulated in the hydrophobic-core PLGA layer, which prevented water molecules from entering the core. As for the shell layer, the molecular structure of DXM contains the element fluorine; it is easy to form a hydrogen bond with the amino group in silk fibroin. The stable combination between them can achieve better sustained release due to the formation of the hydrogen bond. Whereas for the core layer, due to the existence of the Van der Waals force between PLGA and rhBMP2 and the hydrophobicity of PLGA as the core layer, it is not easy for rhBMP2 to pass through the carrier and be rapidly diffused to the shell layer. As a result, the sudden release of rhBMP2 can be well controlled. According to Figure 7, rhBMP2 was released more slowly due to the existence of a core-shell structure. In the sustained 21-day drug release test, the cumulative release rate of DXM is about $50-75 \%$ and the cumulative release rate of rhBMP2 is about 9-28\%.

3.6. Cell Adhesion and Viability Analysis. rBMSCs are widely used in bone tissue engineering, and many scaffolds of implanted cells are used in bone defects. Therefore, rBMSCs were implanted into different groups of materials to detect the ability of cells to proliferate and differentiate. In order to compare the proliferation effect, cover slips were used as blank control. The proliferative activity of the cells analyzed by CCK-8 within 7 days is shown in Figure 8. On the first day after implantation, the proliferation effect of the drugfree fibrous membrane was slightly higher than that of the blank control, which indicated that SF/PLGA can enhance rBMSC proliferation and adhesion $[14,26]$, while the effect of a drug-loaded fibrous membrane on cell proliferation was slightly lower than that of the simple fibrous membrane. Because the burst release of DXM caused toxicity to the cell microenvironment. The drug-loaded fibrous membranes proliferated more compared with those without drug-loaded fibrous membranes on 7 days, suggesting that the addition of DXM and rhBMP2 accelerated the proliferation of rBMSCs. Cell proliferation on the SF-DXM/PLGA fiber membrane was higher than the other groups, verifying that the release of DXM in the early stage played an important role on the cell proliferation. On the 7th day, the proliferation of the SF-DXM/PLGA-rhBMP2 fiber membrane of the cells was much higher than the other groups; rhBMP2 collaborates with DXM in the anaphase promoting the proliferation of rBMSCs. Therefore, the addition of both the DXM and rhBMP2 fibrous membranes has a significant effect on promoting cell growth and proliferation.

Figures 9(a)-9(d) shows SF/PLGA, SF-DXM/PLGA, SF/PLGA-rhBMP2 $(25 \mu \mathrm{g})$, and SF-DXM/PLGA-rhBMP2 $(25 \mu \mathrm{g})$ nanofiber scaffolds 4 days after culturing with rBMSCs. Good cell adhesions were observed. In particular, the rBMSCs that adhered onto SF-DXM/PLGA-rhBMP2 had already stretched along the nanofiber scaffold in all directions. Fluorescence staining images of rBMSCs on nanofiber scaffolds showing morphology and proliferation after 4 days of culture are shown in Figures 10(e)-10(h). The cells had grown and were well spread on and into the nanofibers. 


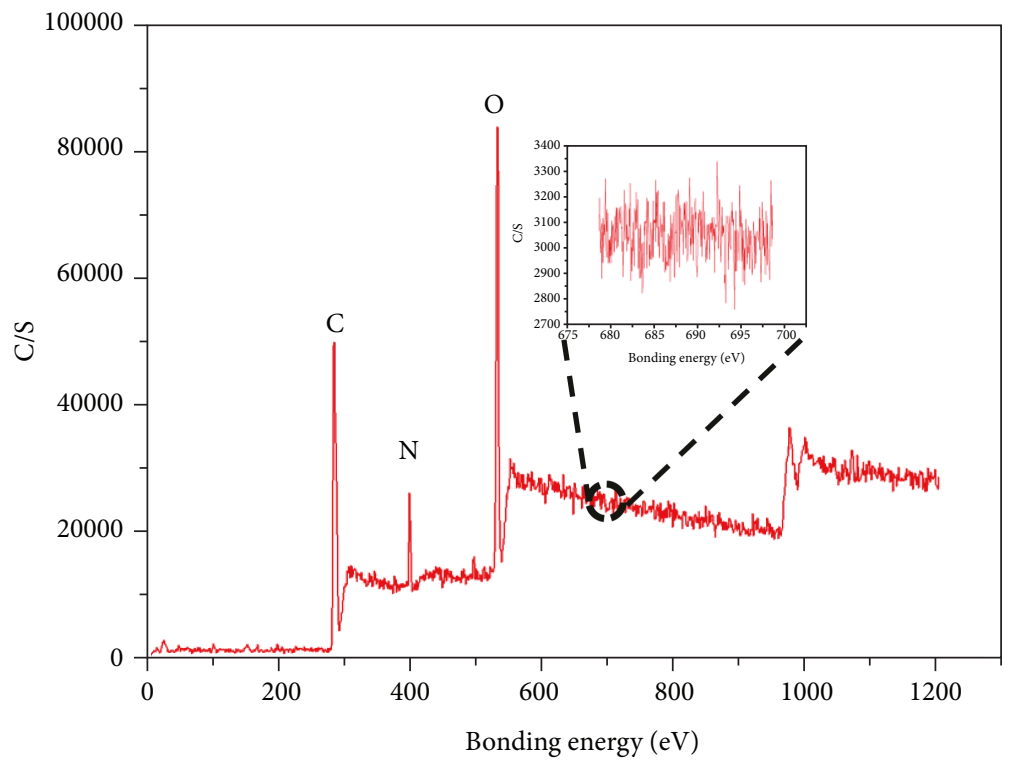

(a)

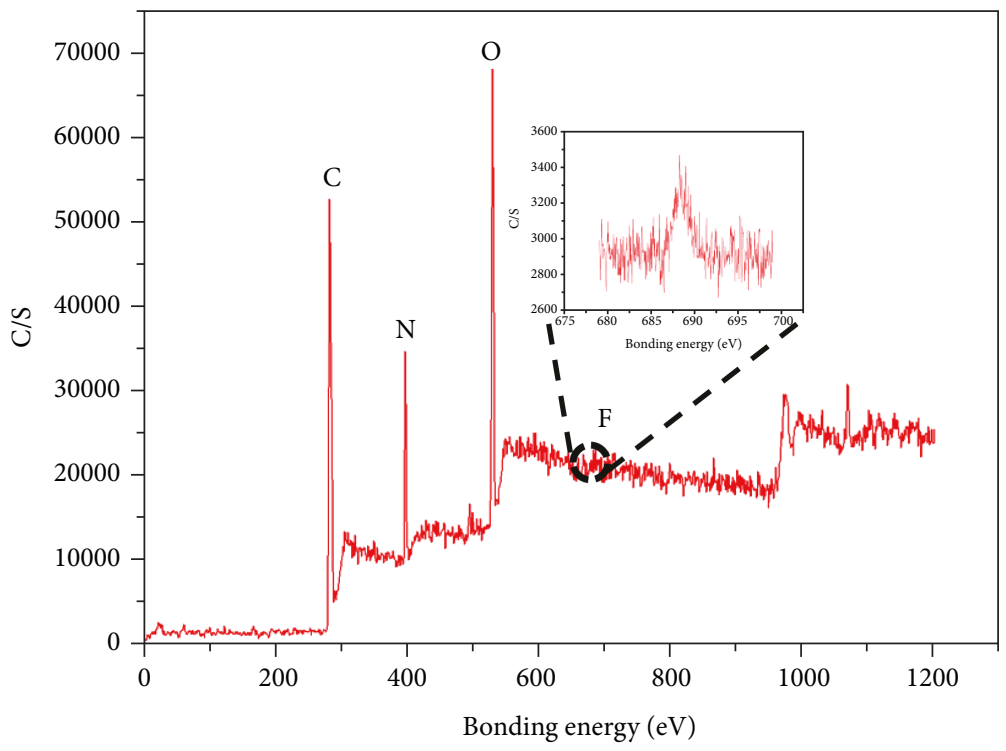

(b)

Figure 5: XPS of the nanofibers: (a) SF/PLGA and (b) SF-DXM/PLGA.

3.7. rBMSC Osteogenic Differentiation. The BCIP/NBT kit was used to determine the ALP activity of rBMSCs [27]. The average optical intensity of the material further characterized the effect of rBMSC differentiation on different groups. When ALP exists, it will catalyze the hydrolysis of BCIP to produce intermediate addition products and further react with NBT to form blue violet crystals. The depth of color can be used to characterize the activity of ALP. The staining results were as shown in Figure 10. Blue-purple staining was observed in all groups. On the third day, ALP staining was light in each group, and the staining of the SF-DXM/PLGA-rhBMP2 $(25 \mu \mathrm{g})$ fibrous membrane group was slightly deep, indicating that the ALP activity of rBMSCs on the scaffold was slightly higher than those of SF/PLGA, SF-DXM/PLGA, and SF/PLGA-rhBMP2 (25 $\mu \mathrm{g})$.
On the 7th day, ALP staining was deeper in each group than on the 3rd day. The ALP staining in SF/PLGA was lower than that in the SF-DXM/PLGA-rhBMP2 (25 $\mu \mathrm{g})$ group. The results showed that nanofiber scaffolds containing both DXM and rhBMP2 drugs could synergistically induce the upregulation of ALP activity in rBMSCs and had better bone induction ability.

Alizarin red reacts with calcium to produce a dark red compound, which stains the calcium nodules deposited [28]. Therefore, ARS was further used to characterized the degree of differentiation. It can be seen from Figure 10 that there is clear positive staining on four groups of fibrous membranes. There are few calcium nodules in the SF/PLGA group, while there are more of those in the SF-DXM/PLGArhBMP2 $(25 \mu \mathrm{g})$ group. These results indicate that the drug- 


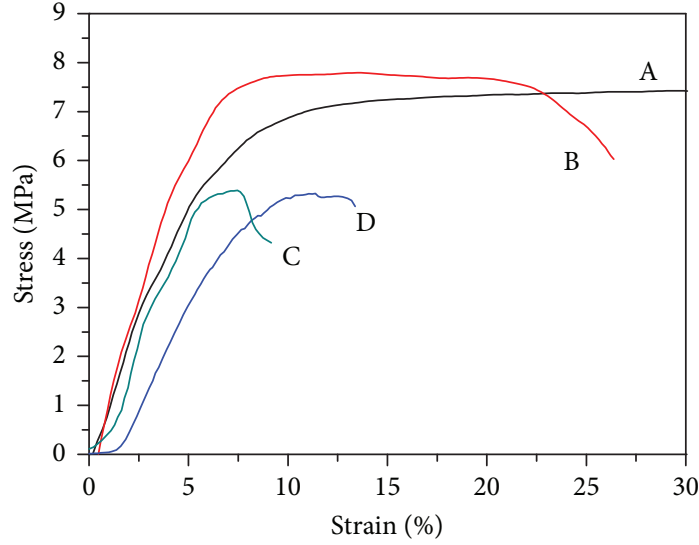

(a)

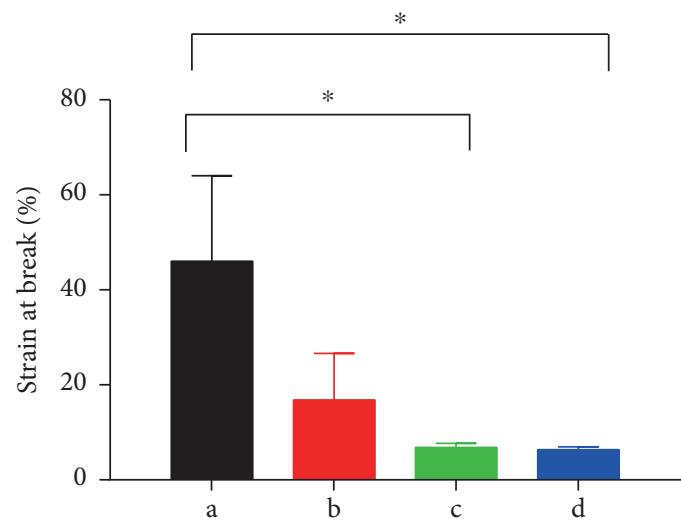

(c)

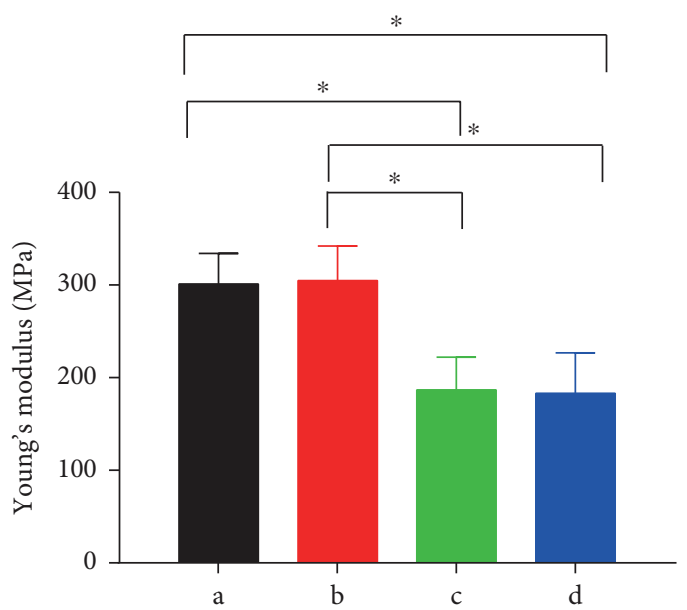

(b)

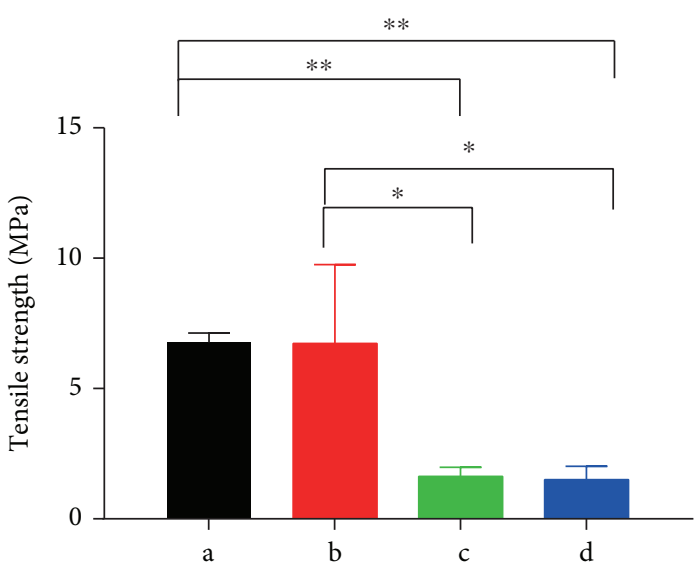

(d)

FIGURE 6: Mechanical properties of an electrospinning nanofiber membrane: (a) typical stress-strain curves, (b) Young's modulus, (c) strain at break, and (d) tensile strength. (a) SF/PLGA, (b) SF-DXM/PLGA, (c) SF/PLGA-rhBMP2, and (d) SF-DXM/PLGA-rhBMP2. ${ }^{*} p<0.05$ and ${ }^{* *} p<0.01$. The content of rhBMP2 is $25 \mu \mathrm{g}$.

loaded coaxial nanofibers are more likely to induce the osteogenic differentiation of rBMSC.

\section{Discussion}

Silk fibroin is the main component of silk fibers. Because of its biocompatibility, nontoxicity, nonirritation, good air permeability, oxygen permeability, and biodegradability, silk fibroin has been attracting interest in the study of drug carrier materials [29]. At present, silk fibroin can be fabricated into various forms, such as membranes [30], microspheres [31], hydrogels [32], porous scaffolds [33], and nanofibers [34]. Among them, the nanofiber membrane prepared by electrospinning technology has become a hotspot in carrier research because of its large specific surface area, high porosity, good cell compatibility, and similar structural characteristics to the natural extracellular matrix (ECM). The silk fibroin membrane prepared in this study is easily soluble in water, and hydrophobic posttreatment is needed if it is used as a drug carrier. Previous studies have shown that the post- treatment of silk fibroin by steam, methanol [35], ethanol, and inorganic aqueous solution [36] is a common method to induce the structural transformation of silk fibroin. The untreated silk fibroin composite membrane exists mainly in a random coil or $\alpha$-helix conformation structure [37], which is soluble in water. The random coil or $\alpha$-helix conformation structure can be transformed to $\beta$-sheets by the treatment of methanol aqueous solution. Silk I and Silk II are two kinds of structures in silk fibroin membranes. The Silk II structure has high stability. Because of random coils and $\alpha$-helix, the Silk I structure is easy to swell under the action of water molecules.

Figure 2 shows the SEM and TEM images of the SF composite membrane fibers. It can be seen that the morphology and orientation of SF composite membrane fibers without drug loading under SEM are better, smoother, more uniformly distributed in diameter, and relatively smaller in diameter (Figure 2(a)). When rhBMP2 was loaded into the nuclear layer, the fibers became flatter and the diameter distribution became wider. The average diameter of the fibers was larger than that of the SF/PLGA group. The reason 

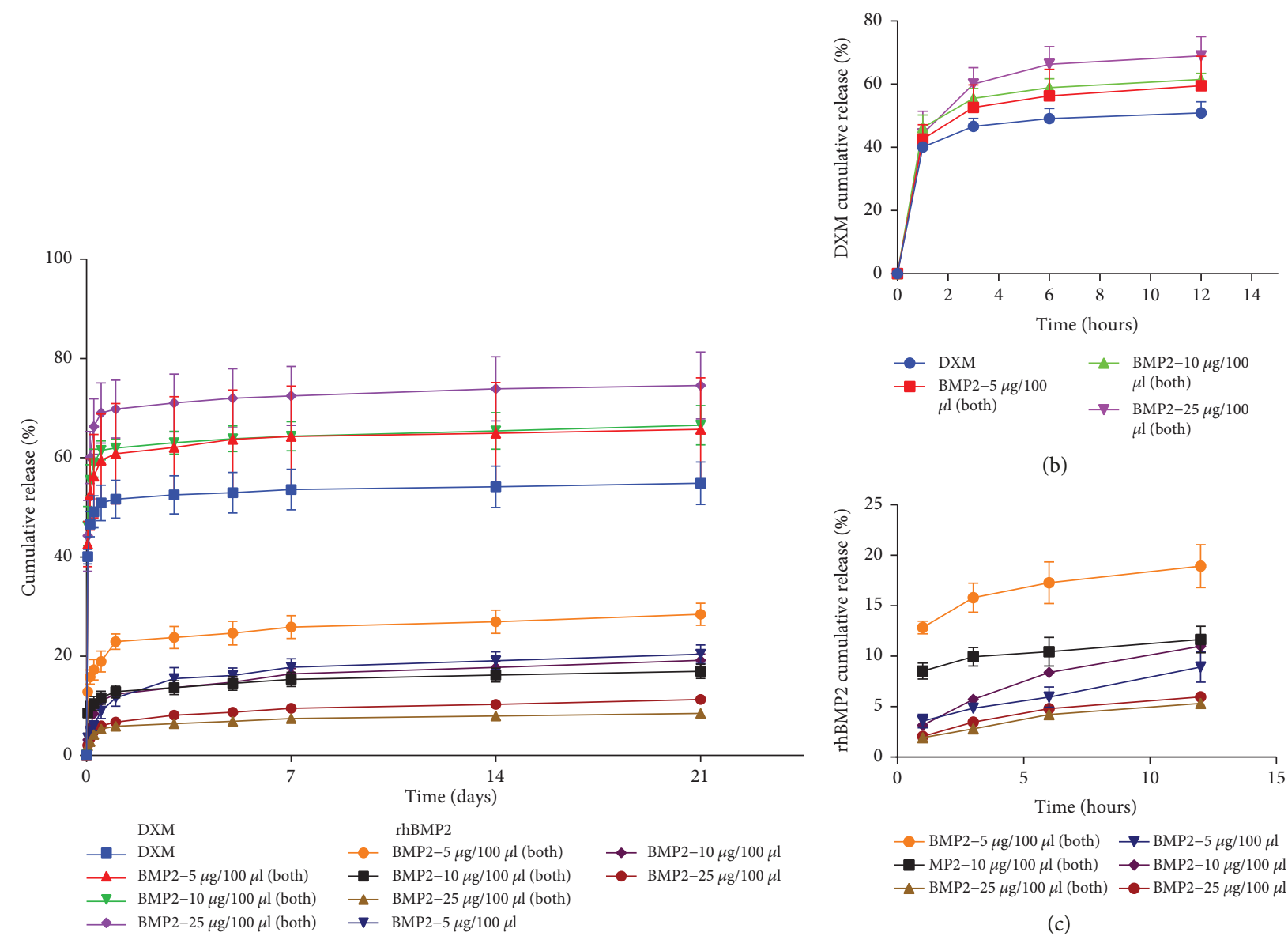

(b)

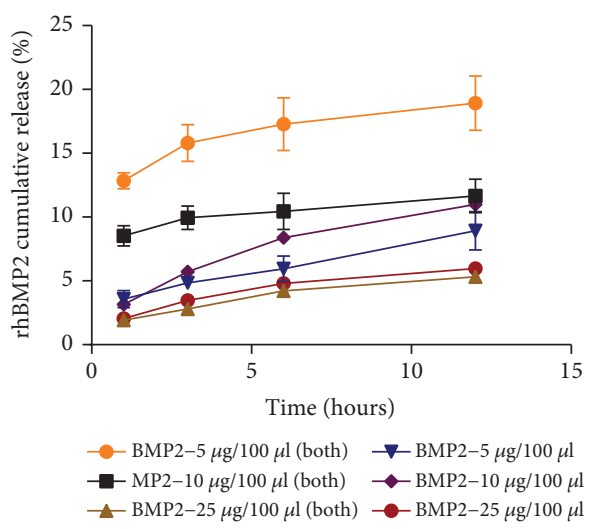

(c)

FIGURE 7: In vitro cumulative release profiles of DXM and rhBMP2: (a) cumulative release curve of drug from different nanofiber membranes in 21 days; (b) cumulative release of DXM from different nanofiber membranes in the initial $12 \mathrm{~h}$; (c) cumulative release of rhBMP2 from different nanofiber mats in the initial $12 \mathrm{~h}$ for both dual-drug-loaded membranes, including DXM and rhBMP2.

may be that rhBMP2 increases the viscosity and surface tension of the PLGA core layer spinning fluid, which makes it more difficult to differentiate the solution under electrostatic force, resulting in the thickening of fibers and poor uniformity (Figures 2(b)-2(d)), and the inner diameter of the PLGA core layer spinning fluid increases from $0.0785 \mu \mathrm{m}$ to $0.136 \mu \mathrm{m}$. However, the difference of rhBMP2 concentration does not show a greater difference in fiber diameter, which may be that the difference of rhBMP2 concentration has less influence on shell solution concentration. When the SF shell solution was added with DXM, the diameter of fibers decreased slightly compared with those of Figures 2(b)-2(d), as shown in Figures 2(e)$2(\mathrm{~g})$. This may be due to the relatively small molecular weight of DXM; when added to the shell spinning solution, the viscosity and surface tension of the spinning solution will decrease. This makes it easier for electrostatic power to overcome surface tension, so the jet solution is easier to differentiate and the diameter of the spinning fiber is slightly smaller than that of SF/PLGA-rhBMP2 $(25 \mu \mathrm{g})$. TEM images show that there is an obvious nuclear layer structure in the fibers, and the nuclear layer structure was stable, and the coreshell boundary was obvious. The ratios of the outer diameter to the inner diameter of SF/PLGA, SF/PLGArhBMP2 $(25 \mu \mathrm{g})$, and SF-DXM/PLGA-rhBMP2 (25 $\mu \mathrm{g})$ are $3.80: 1,4.93: 1$, and $4.80: 1$, respectively.

Hydrophilicity plays an important role in tissue engineering scaffolds, which is helpful for cell infiltration and adhesion [38]. Although PLGA has good biological properties, its poor hydrophilicity limits its wide application. In order to improve the hydrophilicity of PLGA, SF which has better hydrophilicity is introduced through coaxial electrospinning technology. From Figure 3, it is indicated that the hydrophobic nature of the PLGA scaffold can be modified ideally by introducing SF. It is helpful to promote cell adhesion.

In the infrared spectrum of the SF/PLGA nanofiber film (Figure 4), there are both characteristic absorption peaks of SF and PLGA, and the position of the absorption peaks is basically not shifted, which indicates that the interaction force between them is weak. Fourier-transform infrared spectroscopy (FTIR) is one of the common methods to study the secondary structure of silk fibroin. Therefore, the silk fibroin membrane treated with methanol was studied by FTIR. In Figure 4, the regions of amide I $\left(1600-1700 \mathrm{~cm}^{-1}\right)$ and amide II $\left(1500-1600 \mathrm{~cm}^{-1}\right)$ were selected to monitor the formation 


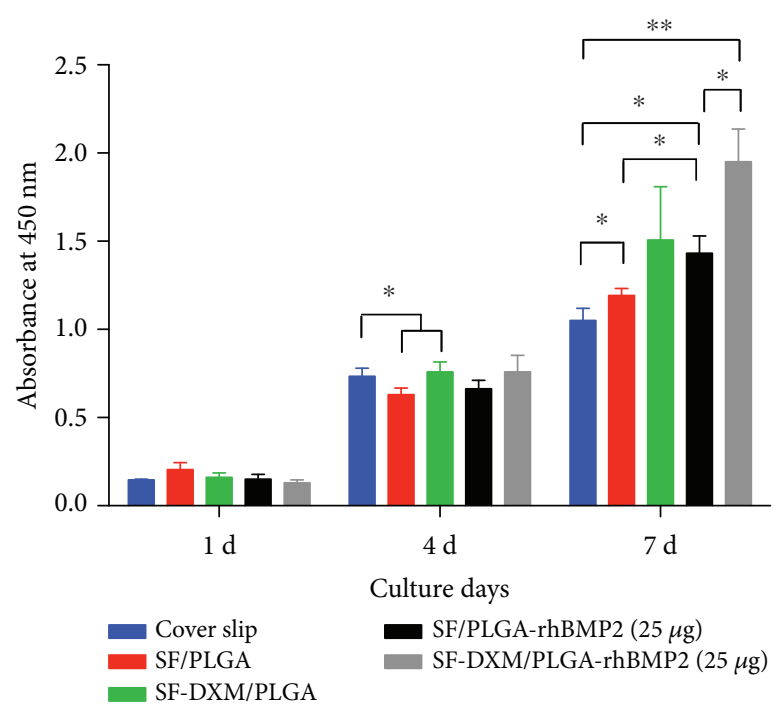

Figure 8: Proliferation of mesenchymal stem cells 1,4 , and 7 days after seeding on various groups, as measured by CCK- 8 assay. ${ }^{*} p<0.05$ and ${ }^{* *} p<0.01$.

of $\beta$-folding structures. In the FTIR spectra of silk fibroin, $1655 \pm 5 \mathrm{~cm}^{-1}$ and $1545 \pm 5 \mathrm{~cm}^{-1}$ are random coil or $\alpha$-helix structures, and $1515 \pm 5 \mathrm{~cm}^{-1}$ is a $\beta$-fold structure [39]. The absorption peaks of SF were mainly around $1660 \mathrm{~cm}^{-1}$ (amide I) and $1530 \mathrm{~cm}^{-1}$ (amide II) without methanol solution treatment, which indicated that SF was mainly a random coil. When SF fibers were treated with methanol aqueous solution, the characteristic absorption peaks of the amide II region gradually changed from $1530 \mathrm{~cm}^{-1}$ to $1519 \mathrm{~cm}^{-1}$, indicating that there was a transition from a random coil to a $\beta$-fold structure in the process of methanol aqueous solution treatment.

Figure 6 is an analysis of the mechanical properties of fiber membranes. The results show that there was a great influence on the mechanical properties of fiber membranes with the addition of rhBMP2. Because the PLGA solution in the core layer is loaded with rhBMP2, the solution viscosity increases, and the difficulty of solution differentiation increases under the action of the electrostatic force; the stability of electrospinning is also affected, resulting in fiber diameter distribution extending to $0.2-1.2 \mu \mathrm{m}$, while the diameter distribution of fibers in the pure SF/PLGA membrane is in the range of $0.1-0.8 \mu \mathrm{m}$. The widening of the distribution makes the force of the fiber membrane uneven.

In vitro release curves of rhBMP2 and DXM from the drug-loaded nanofiber membrane at $37^{\circ} \mathrm{C}$ within 21 days are shown in Figure 7. In the sustained release system of the SF membrane, DXM can be distributed not only in the amorphous region but also between the $\beta$ lamellae in the crystalline region. When water molecule permeation loosens its skeleton, the drug dissolves under the action of the concentration gradient, which is retarded by the polypeptide chain of the amorphous region and Silk II crystallization. However, although the samples treated with methanol aqueous solution contain different degrees of the Silk II structure, water molecules still penetrate into the Silk I structure of the fibers, causing the swelling of the fibers or forming voids in the adjacent amorphous region to promote drug diffusion [40], which also causes the sudden release of DXM in the SF/PLGA composite membrane.

When rhBMP2 is loaded into the nanofiber membrane, it can be found that DXM, as the shell layer, releases faster with the increase of the rhBMP2 content in the core layer. With the increase of rhBMP2 concentration, the viscosity of the nuclear solution increases, which enhances the intermolecular force between PLGA and BMP2, weakens the effect of PLGA on the small molecule DXM, reduces the resistance of DXM to release outward, and shows the result of the faster release rate of DXM.

On the contrary, PLGA as the core structure is a hydrophobic material, which prevents water molecules from entering the core structure; rhBMP2 is released from core layer to the shell layer first and is then diffused by the shell layer. Therefore, the release of rhBMP2 was slower and more sustained, and with the increase of the rhBMP2 concentration, the release rate of rhBMP2 decreased within the similar diameter of the fibers.

DXM plays an important role in promoting the proliferation and differentiation of rBMSCs. Therefore, DXM is conducive to cell proliferation when released in large quantities at an early stage, and rhBMP2 accumulates more after seven days, which is conducive to the osteogenic differentiation of rBMSCs. In addition, the synergistic effect of DXM and rhBMP2 is more conducive to the osteogenic differentiation of rBMSCs in the middle and late stages.

Therefore, we should choose a concentration combination of a faster DXM release and a slower rhBMP2 release for in vitro experiments; according to in vitro drug release results, we see that when the rhBMP2 concentration is $25 \mu \mathrm{g} / 100 \mu \mathrm{l}, \mathrm{DXM}$ is released faster and rhBMP2 is released slower than the other groups. So we chose the SF-DXM/PLGA-rhBMP2 $(25 \mu \mathrm{g} / 100 \mu \mathrm{l})$ group for the next experiment. Figure 8 shows the cck- 8 detection results of rBMSCs on four groups of the composite nanofiber 


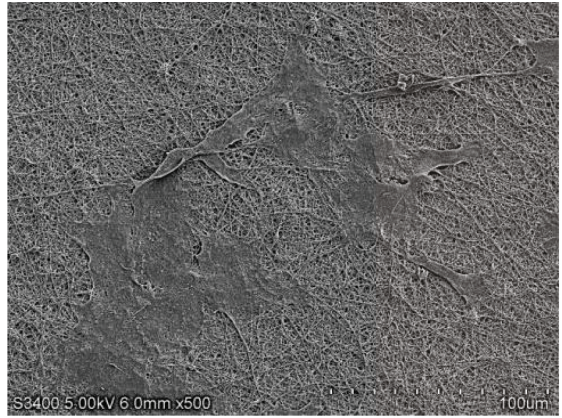

(a)

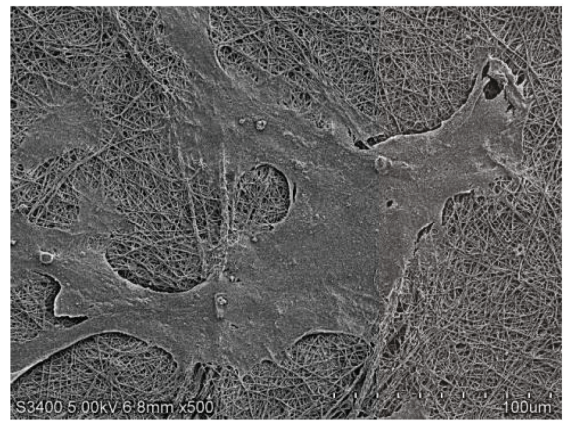

(c)

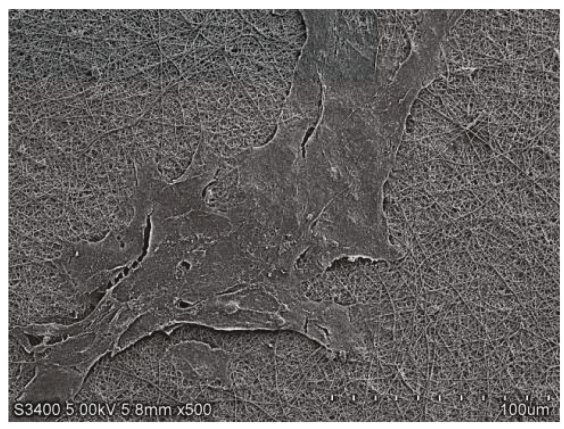

(e)

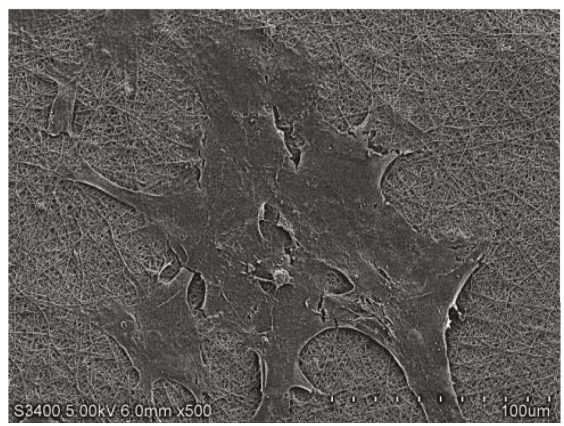

(g)

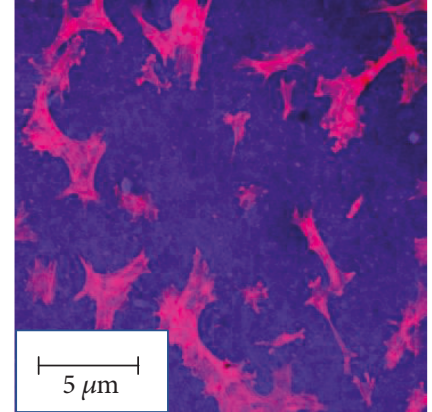

SF/PLGA

(b)

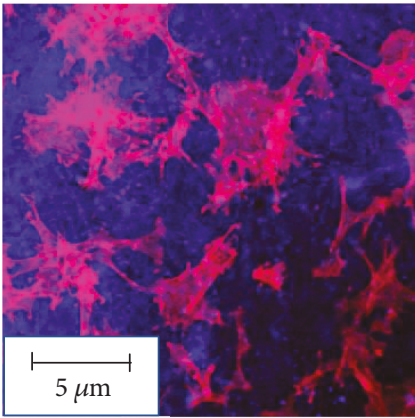

(d)

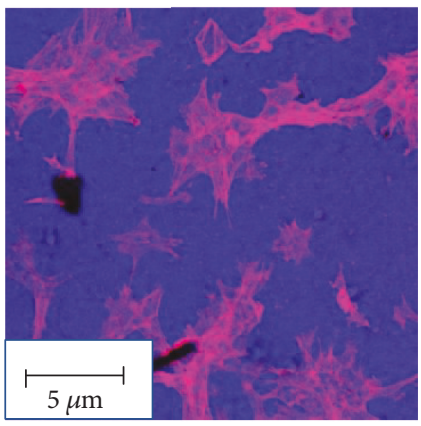

(f)

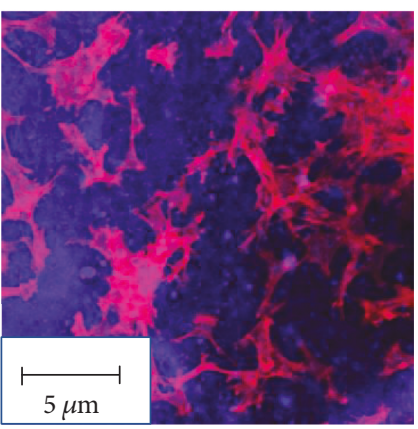

SF-DXM/PLGA-rhBMP2

$(25 \mu \mathrm{g})$

(h)

SF-DXM/PLGA

SF/PLGA-rhBMP2 $(25 \mu \mathrm{g})$

Figure 9: SEM images of adhered rBMSCs after 4 days of culture on (a) SF/PLGA, (b) SF-DXM/PLGA, (c) SF/PLGA-rhBMP2, and (d) SFDXM/PLGA-rhBMP2. Scale bar $=100 \mu \mathrm{m}$. Fluorescence staining of rBMSCs after 4 days of culture on (e) SF/PLGA, (f) SF-DXM/PLGA, (g) SF/PLGA-rhBMP2, and (h) SF-DXM/PLGA-rhBMP2. Red is F-actin, blue is nucleus. Scale bar $=5 \mu \mathrm{m}$.

membrane. In total, the cell activity on the fibrous membrane increased with time in four groups within 7 days. The cell activity in the SF-DXM/PLGA group within 4 days was larger than the SF/PLGA and SF/PLGA-rhBMP2 (25 $\mu \mathrm{g})$ group; there was no significant difference between these two groups. The cell activity on the SF-DXM/PLGA-rhBMP2 (25 $\mu \mathrm{g})$ group replaced that of the SF-DXM/PLGA group as the largest within 4 to 7 days, and the cell activity in the TCP group 


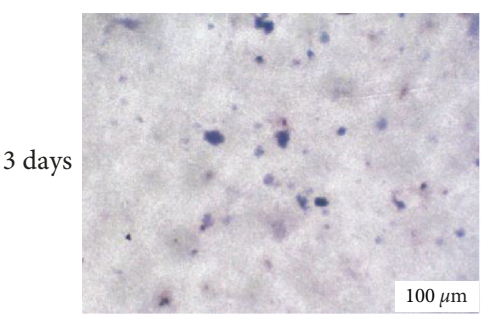

(a)

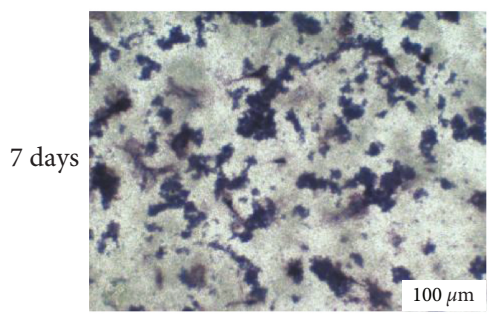

(e)

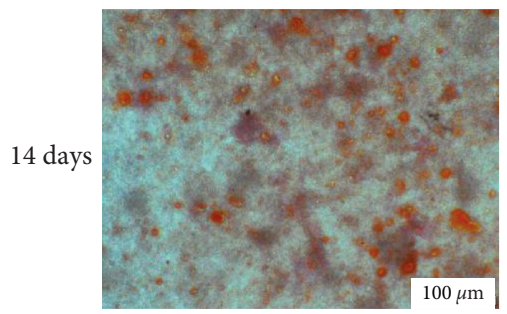

(i)

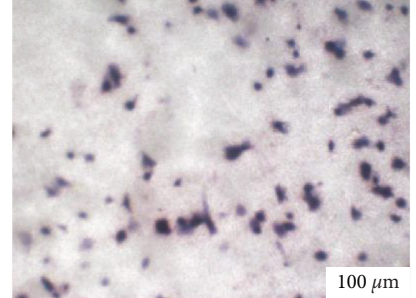

(b)

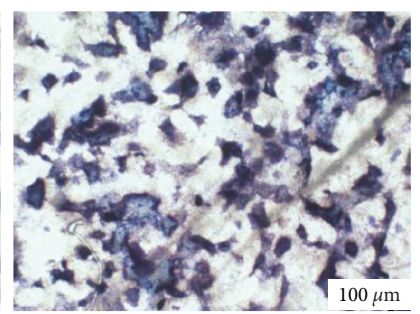

(f)

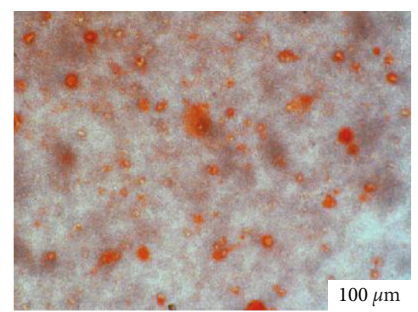

(j)

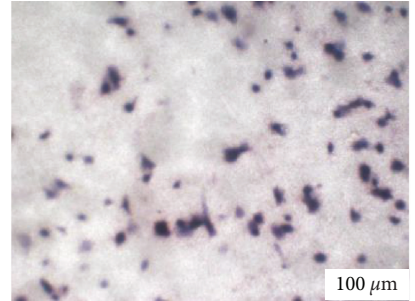

(c)

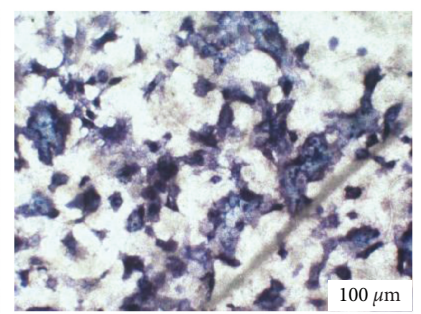

(g)

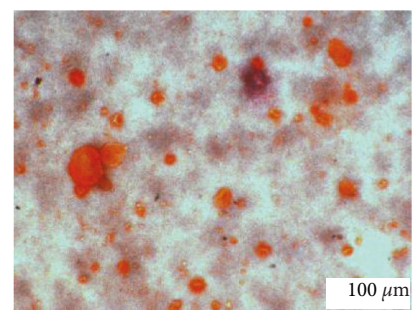

(k)

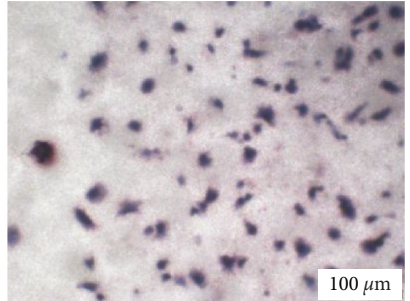

(d)

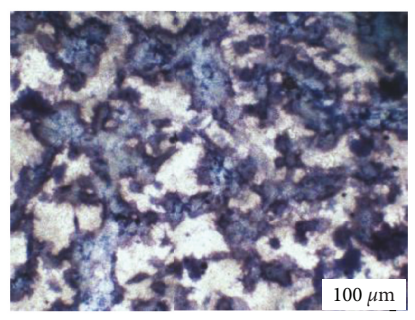

(h)

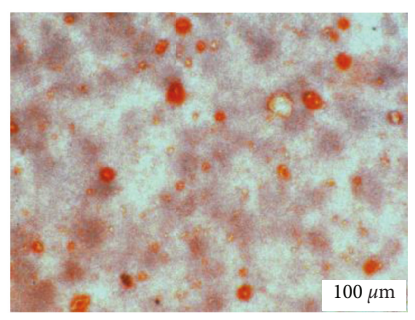

(1)

FIGURe 10: Representative staining of ALP on day 3: (a) SF/PLGA, (b) SF-DXM/PLGA, (c) SF/PLGA-rhBMP2, and (d) SF-DXM/PLGArhBMP2. Representative staining of ALP on day 7: (e) SF/PLGA, (f) SF-DXM/PLGA, (g) SF/PLGA-rhBMP2, and (h) SF-DXM/PLGArhBMP2. Representative staining of ARS on day 14: (i) SF/PLGA, (j) SF-DXM/PLGA, (k) SF/PLGA-rhBMP2, and (l) SF-DXM/PLGArhBMP2. The content of rhBMP2 is $25 \mu \mathrm{g}$. Scale bar $=100 \mu \mathrm{m}$.

was the smallest. The reasons for the above results may be the sudden release of a large amount of DXM in the SFDXM/PLGA group and the SF-DXM/PLGA-rhBMP 2 $(25 \mu \mathrm{g})$ group within 4 days. After 7 days, with the cell fluid exchanged and the release of DXM decreased, the release of rhBMP2 gradually increased. So the activity of the SFDXM/PLGA-rhBMP2 $(25 \mu \mathrm{g})$ group was the highest. There was no drug release in the TCP group and the SF/PLGA group, but the activity of the cells in the TCP group was lower than that in SF/PLGA group. It can be concluded that the existence of pores in the fiber membrane enables the normal passage of nutrients inside and outside the membrane, while the release of DXM and rhBMP2 inside the fiber membrane, especially the slow and long-term release, promotes the growth and reproduction of rBMSCs around and on the surface of the fiber membrane.

It can be seen from Figure 9 that after 4 days of growth, rBMSCs were planted on the surface of the fibrous membrane, and the cells adhered to each other or the fibrous membrane, showing a spreading trend, and the four groups of fibrous membrane could be considered to have good biocompatibility.

Figure 10 shows the results of ALP staining and ARS staining of the fibrous membrane in each group. In ALP staining, the color of the four groups was significantly darker on the seventh day than on the third day, indicating that the ALP activity of the four groups showed an increasing trend within 7 days, and the SF-DXM/PLGA-rhBMP2 $(25 \mu \mathrm{g})$ group was significantly darker than that of the other groups on the seventh day. The staining of the SFDXM/PLGA group and the SF/PLGA-rhBMP2 $(25 \mu \mathrm{g})$ group was deeper than that of the SF/PLGA group. ALP staining can, to a certain extent, reflect the release of the drug loaded in the fiber membrane. The massive release of drug-loaded fibrous membranes in the three groups within 3 days, especially DXM, effectively stimulated the improvement of ALP activity. However, with the gradual increase of rhBMP2 release after 3 days, the ALP activity of the SF/PLGArhBMP2 $(25 \mu \mathrm{g})$ and SF-DXM/PLGA-rhBMP2 $(25 \mu \mathrm{g})$ groups also increased. Therefore, at 7 days, the ALP activity of the SF-DXM/PLGA-rhBMP2 $(25 \mu \mathrm{g})$ group was the highest, while the ALP activity of the SF/PLGA group was always at a low level due to the absence of drug release in the SF/PLGA group. It also can be seen from Figure 10 that there is a clear positive staining on the four groups of fibrous membranes. There are few calcium nodules in the SF/PLGA group, while there are more of those in the SF-DXM/PLGA-rhBMP2 $(25 \mu \mathrm{g})$ group. These results indicate that the drug-loaded coaxial nanofibers are more likely to induce osteogenic differentiation of rBMSCs. 


\section{Conclusion}

The SF/PLGA nanofiber membrane was successfully fabricated for the sustained delivery of rhBMP2 and DXM by coaxial electrospinning for bone tissue engineering. Our results showed that coaxial fibrous membranes with dualdrug loading can improve the adhesion, diffusion, and proliferation of rBMSCs. More importantly, rhBMP2 and DXM can play a synergistic role in promoting the osteogenic differentiation of stem cells even under nonosteoinductive conditions. In summary, these functional nanocomposites have broadened the number of choices of scaffolds to be potentially used in bone tissue engineering applications.

\section{Data Availability}

The data used to support the findings of this study are available from the corresponding author upon request.

\section{Conflicts of Interest}

The authors declare that there is no conflict of interest regarding the publication of this paper.

\section{Acknowledgments}

The work was supported by Jilin Province Health Project 2018 (3D517EB73428).

\section{References}

[1] T. T. Roberts and A. J. Rosenbaum, "Bone grafts, bone substitutes and orthobiologics: the bridge between basic science and clinical advancements in fracture healing," Organogenesis, vol. 8, no. 4, pp. 114-124, 2014.

[2] S. P. Nukavarapu, S. G. Kumbar, J. L. Brown et al., "Polyphosphazene/nano-hydroxyapatite composite microsphere scaffolds for bone tissue engineering," Biomacromolecules, vol. 9, no. 7, pp. 1818-1825, 2008.

[3] N. L. Morozowich, J. L. Nichol, and H. R. Allcock, "Investigation of apatite mineralization on antioxidant polyphosphazenes for bone tissue engineering," Chemistry of Materials, vol. 24, no. 17, pp. 3500-3509, 2012.

[4] S. Hofmann, H. Hagenmüller, A. M. Koch et al., "Control of in vitro tissue-engineered bone-like structures using human mesenchymal stem cells and porous silk scaffolds," Biomaterials, vol. 28, no. 6, pp. 1152-1162, 2007.

[5] A. Di Martino, L. Liverani, A. Rainer, G. Salvatore, M. Trombetta, and V. Denaro, "Electrospun scaffolds for bone tissue engineering," Musculoskeletal Surgery, vol. 95, no. 2, pp. 69-80, 2011.

[6] X. Gao, X. Zhang, J. Song et al., "Osteoinductive peptidefunctionalized nanofibers with highly ordered structure as biomimetic scaffolds for bone tissue engineering," International Journal of Nanomedicine, vol. 10, pp. 7109-7128, 2015.

[7] J. M. Holzwarth and P. X. Ma, "Biomimetic nanofibrous scaffolds for bone tissue engineering," Biomaterials, vol. 32, no. 36, pp. 9622-9629, 2011.

[8] Z. X. Meng, W. Zheng, L. Li, and Y. F. Zheng, "Fabrication, characterization and in vitro drug release behavior of electro- spun PLGA/chitosan nanofibrous scaffold," Materials Chemistry and Physics, vol. 125, no. 3, pp. 606-611, 2011.

[9] Y. Wan, X. Qu, J. Lu et al., "Characterization of surface property of poly(lactide-co-glycolide) after oxygen plasma treatment," Biomaterials, vol. 25, no. 19, pp. 4777-4783, 2004.

[10] L. Kong and G. R. Ziegler, "Molecular Entanglement and Electrospinnability of Biopolymers," Journal of Visualized Experiments, vol. 2014, no. 91, article e51933, 2014.

[11] L. Li, H. Li, Y. Qian et al., "Electrospun poly ( $\varepsilon$-caprolactone)/silk fibroin core-sheath nanofibers and their potential applications in tissue engineering and drug release," International Journal of Biological Macromolecules, vol. 49, no. 2, pp. 223232, 2011.

[12] X. Yang, L. Fan, L. Ma et al., "Green electrospun Manuka honey/silk fibroin fibrous matrices as potential wound dressing," Materials \& Design, vol. 119, pp. 76-84, 2017.

[13] M. Li, M. Ogiso, and N. Minoura, "Enzymatic degradation behavior of porous silk fibroin sheets," Biomaterials, vol. 24, no. 2, pp. 357-365, 2003.

[14] J. Melke, S. Midha, S. Ghosh, K. Ito, and S. Hofmann, "Silk fibroin as biomaterial for bone tissue engineering," Acta Biomaterialia, vol. 31, pp. 1-16, 2016.

[15] Y. Lu, J. Huang, G. Yu et al., "Coaxial electrospun fibers: applications in drug delivery and tissue engineering," Wiley Interdisciplinary Reviews: Nanomedicine and Nanobiotechnology, vol. 8, no. 5, pp. 654-677, 2016.

[16] X. Zhao, Y. Lui, P. Toh, and S. Loo, "Sustained release of hydrophilic L-ascorbic acid 2-phosphate magnesium from electrospun polycaprolactone scaffold-a study across blend, coaxial, and emulsion electrospinning techniques," Materials, vol. 7, no. 11, pp. 7398-7408, 2014.

[17] P. McClellan and W. J. Landis, "Recent applications of coaxial and emulsion electrospinning methods in the field of tissue engineering," BioResearch Open Access, vol. 5, no. 1, pp. 212 227, 2016.

[18] B.-R. Kim, T. B. L. Nguyen, Y.-K. Min, and B.-T. Lee, "In vitro and in vivo studies of BMP-2-loaded PCL-gelatin-BCP electrospun scaffolds," Tissue Engineering. Part A, vol. 20, no. 23-24, pp. 3279-3289, 2014.

[19] C. Del Rosario, M. Rodriguez-Evora, R. Reyes et al., "Evaluation of nanostructure and microstructure of bone regenerated by BMP-2-porous scaffolds," Journal of Biomedical Materials Research. Part A, vol. 103, no. 9, pp. 2998-3011, 2015.

[20] P. Boyne and S. D. Jones, "Demonstration of the osseoinductive effect of bone morphogenetic protein within endosseous dental implants," Implant Dentistry, vol. 13, no. 2, pp. 180184, 2004.

[21] J. M. Wozney, "The bone morphogenetic protein family and osteogenesis," Molecular Reproduction and Development, vol. 32, no. 2, pp. 160-167, 1992.

[22] S. Fuchigami, T. Nakamura, K. Furue, K. Sena, Y. Shinohara, and K. Noguchi, "Recombinant human bone morphogenetic protein-9 potently induces osteogenic differentiation of human periodontal ligament fibroblasts," European Journal of Oral Sciences, vol. 124, no. 2, pp. 151-157, 2016.

[23] C. M. Verhoef, J. A. G. van Roon, M. E. Vianen, F. P. J. G. Lafeber, and J. W. J. Bijlsma, "The immune suppressive effect of dexamethasone in rheumatoid arthritis is accompanied by upregulation of interleukin 10 and by differential changes in interferon $\gamma$ and interleukin 4 production," Annals of the Rheumatic Diseases, vol. 58, no. 1, pp. 49-54, 1999. 
[24] M. Zhao, P. Li, H. Xu et al., "Dexamethasone-activated MSCs release MVs for stimulating osteogenic response," Stem Cells International, vol. 2018, Article ID 7231739, 12 pages, 2018.

[25] M. Yuasa, T. Yamada, T. Taniyama et al., "Dexamethasone enhances osteogenic differentiation of bone marrow- and muscle-derived stromal cells and augments ectopic bone formation induced by bone morphogenetic protein-2," PLoS One, vol. 10, no. 2, article $\mathrm{e} 0116462,2015$.

[26] G. Wang, H. Yang, M. Li, S. Lu, X. Chen, and X. Cai, “The use of silk fibroin/hydroxyapatite composite co-cultured with rabbit bone-marrow stromal cells in the healing of a segmental bone defect," The Journal of Bone and Joint Surgery. British volume, vol. 92-B, no. 2, pp. 320-325, 2010.

[27] K. T. Shalumon, G. J. Lai, C. H. Chen, and J. P. Chen, "Modulation of bone-specific tissue regeneration by incorporating bone morphogenetic protein and controlling the shell thickness of silk fibroin/chitosan/nanohydroxyapatite core-shell nanofibrous membranes," ACS Applied Materials \& Interfaces, vol. 7, no. 38, pp. 21170-21181, 2015.

[28] S. Sancilio, M. Gallorini, C. di Nisio et al., "Alginate/hydroxyapatite-based nanocomposite scaffolds for bone tissue engineering improve dental pulp biomineralization and differentiation," Stem Cells International, vol. 2018, Article ID 9643721, 13 pages, 2018.

[29] C. Marcolin, L. Draghi, M. Tanzi, and S. Fare, "Electrospun silk fibroin-gelatin composite tubular matrices as scaffolds for small diameter blood vessel regeneration," Journal of Materials Science: Materials in Medicine, vol. 28, no. 5, 2017.

[30] F. P. Seib and D. L. Kaplan, "Doxorubicin-loaded silk films: drug-silk interactions and in vivo performance in human orthotopic breast cancer," Biomaterials, vol. 33, no. 33, pp. 8442-8450, 2012.

[31] Z. Zhong and J. Ma, "Fabrication, characterization, and in vitro study of zinc substituted hydroxyapatite/silk fibroin composite coatings on titanium for biomedical applications," Journal of Biomaterials Applications, vol. 32, no. 3, pp. 399409, 2017.

[32] C. H. Park, L. Jeong, D. Cho, O. H. Kwon, and W. H. Park, "Effect of methylcellulose on the formation and drug release behavior of silk fibroin hydrogel," Carbohydrate Polymers, vol. 98, no. 1, pp. 1179-1185, 2013.

[33] N. Kasoju and U. Bora, "Fabrication and characterization of curcumin-releasing silk fibroin scaffold," Journal of Biomedical Materials Research Part B: Applied Biomaterials, vol. 100B, no. 7, pp. 1854-1866, 2012.

[34] X. Sheng, L. Fan, C. He, K. Zhang, X. Mo, and H. Wang, "Vitamin E-loaded silk fibroin nanofibrous mats fabricated by green process for skin care application," International Journal of Biological Macromolecules, vol. 56, pp. 49-56, 2013.

[35] X. Hu, K. Shmelev, L. Sun et al., "Regulation of silk material structure by temperature-controlled water vapor annealing," Biomacromolecules, vol. 12, no. 5, pp. 1686-1696, 2011.

[36] G. Lu, S. Liu, S. Lin, D. L. Kaplan, and Q. Lu, "Silk porous scaffolds with nanofibrous microstructures and tunable properties," Colloids and Surfaces B: Biointerfaces, vol. 120, pp. 28-37, 2014.

[37] Z. He, Z. Liu, X. Zhou, and H. Huang, "Low pressure-induced secondary structure transitions of regenerated silk fibroin in its wet film studied by time-resolved infrared spectroscopy," Proteins: Structure, Function, and Bioinformatics, vol. 86, no. 6, pp. 621-628, 2018.
[38] B. D. Boyan, E. M. Lotz, and Z. Schwartz, "Roughness and Hydrophilicity as Osteogenic Biomimetic Surface Properties," Tissue Engineering Part A, vol. 23, no. 23-24, pp. 1479-1489, 2017.

[39] X. Xie, L. Liu, Z. Zheng et al., "Silk fibroin-based fibrous anal fistula plug with drug delivery function," Macromolecular Bioscience, vol. 18, no. 4, article 1700384, 2018.

[40] M. Choi, D. Choi, and J. Hong, "Correction to "Multilayered controlled drug release silk fibroin nanofilm by manipulating secondary structure"," Biomacromolecules, vol. 19, no. 9, pp. 3902-3903, 2018. 


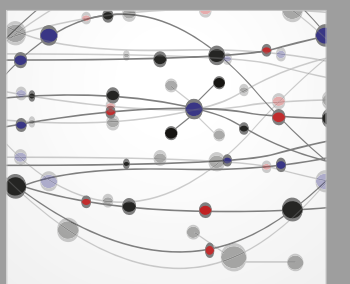

The Scientific World Journal
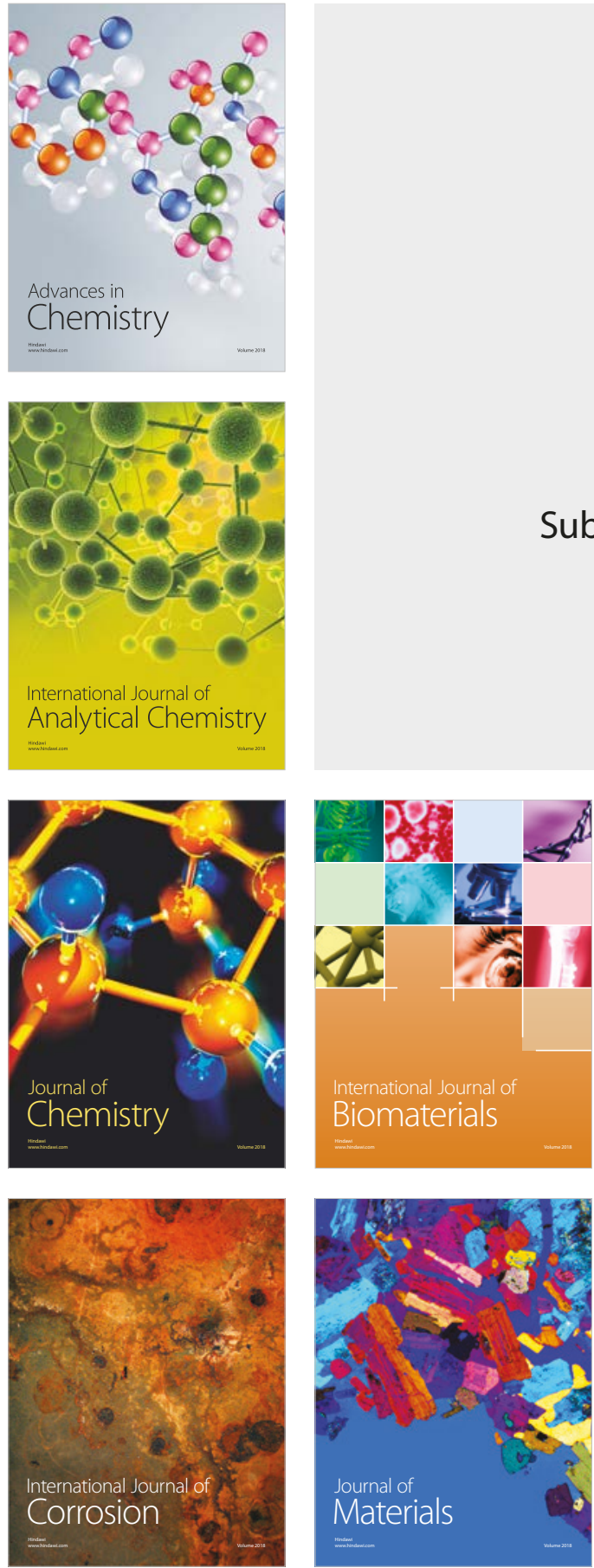

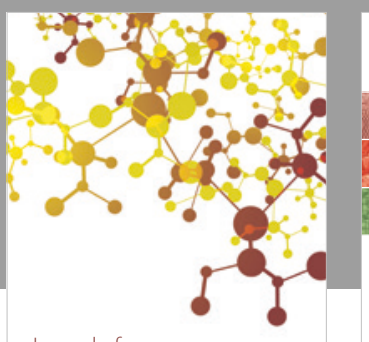

Journal of

Applied Chemistry
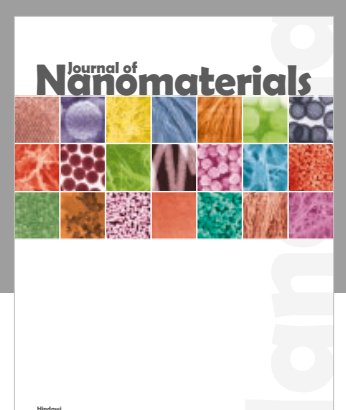

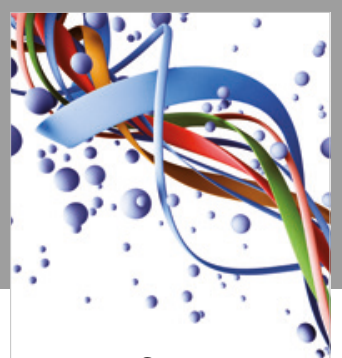

Scientifica

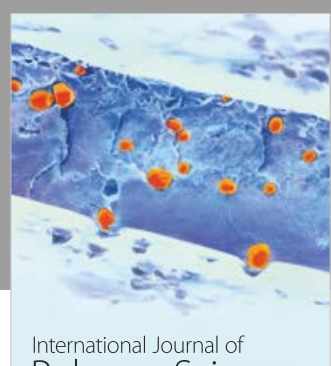

Polymer Science

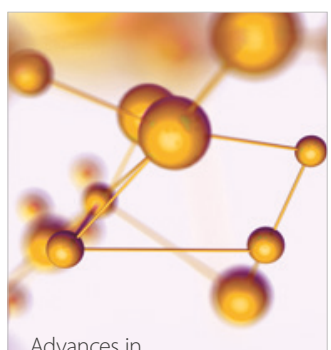

Physical Chemistry
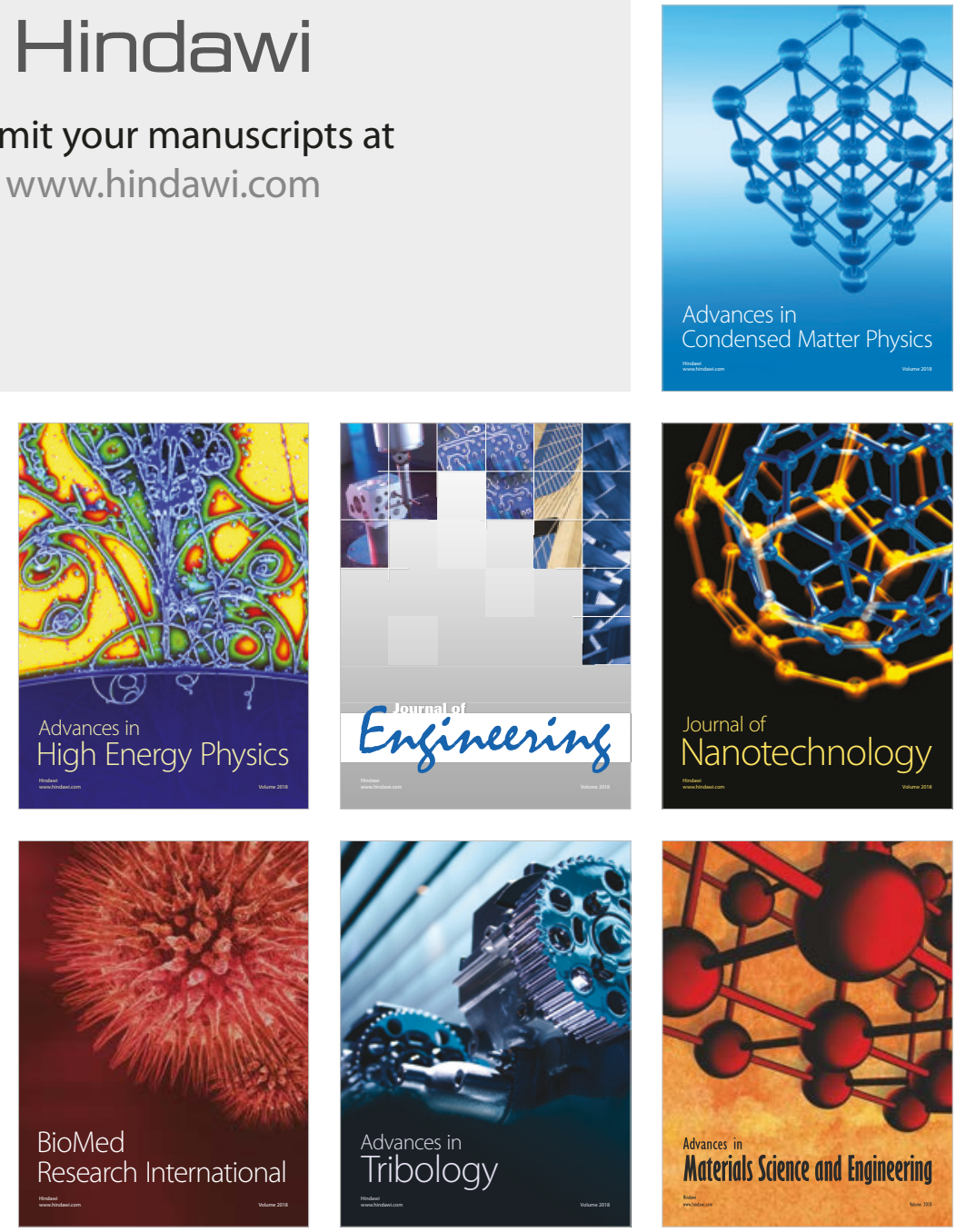